\title{
ESTUDO DE FUNÇÕES QUE SE AJUSTAM À MATURAÇÃO DA CANA-DE-AÇÚCAR
}

\author{
MARGARETH C. TOYAMA UDO
}

Bel. Matemática

Orientador: Prof. Dr. ROBERTO S. MORAES

Dissertação apresentada à Escola Superior de Agricultura "Luiz de Queiroz", da Universidade de São Paulo, para obtenção do Título de Mestre em Agronomia - Área de Concentração Experimentação $\theta$ Estatistica.

$P I R A C I C A B A$

Estado de São Paulo - Brasil

Maio - 1983 
ii.

A Deus

\author{
A meus pais, \\ meus irmãos e \\ meu esposo
}

DEDICO. 


\section{AGRADECIMENTOS}

Ao Professor Dr. Roberto Simionato Moraes, pela orientação segura e valiosa durante todo o curso e, em especial, na realização deste trabalho.

Aos Professores do Departamento de Matemática e Estatistí ca da Escola Superior de Agricultura "Luiz de Queiroz", USP, pelos ensinamentos transmitidos.

Ao Professór Dr. Antonio Francisco Iemma pela revisão e pelas valiosas sugestões apresentadas na realização deste trabalho.

Ao Professor Dr. Vivaldo Francisco da Cruz pelas valiosas sugestões apresentadas na realização deste trabalho.

Aos colegas Eliza Helena de Souza Faria e Elio Paulo Zanta, pela amizade e troca de idéias durante o curso.

Aos demais colegas do Curso de Pós-Graduação, pelo excelente convívio durante o curso.

Ao meu esposo, Paulo, pelo apoio e incentivo.

Aos colegas do Departamento de Matemática e Estatística, da Universidade Estadual de Maringá, pelo incentivo e colaboração.

Ao CNPq, pelo auxílio financeiro prestado.

Aos funcionários do Centro de Processamento de Dados, da Escola Superior de Agricultura "Luiz de Queiroz", USP especialmente a walter João Dihel pela constante cola boração. 
A Maria Izalina Ferreira Alves, Rosa Maria Alves, Otávio Frasseto e Maria Alayde P. de Souza pela amizade.

Ao Professor Dr. Cássio R. de Melo Godói pela versão do resumo. 


\section{INDICE}

Pāgina

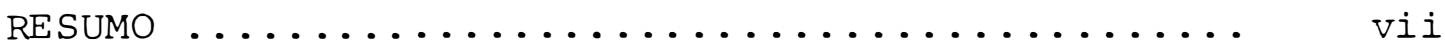

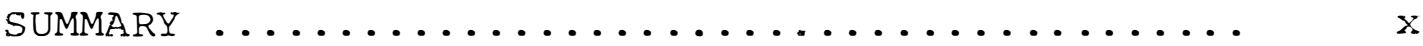

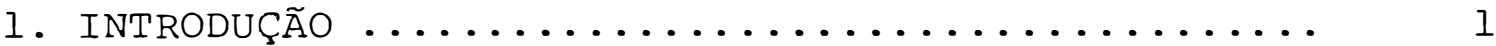

1.1. Segunda Aproximação de Mitscherlich ....... 2

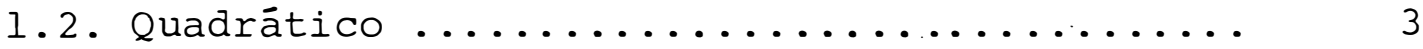

1.3. Raíz Quadrada ..................... 3

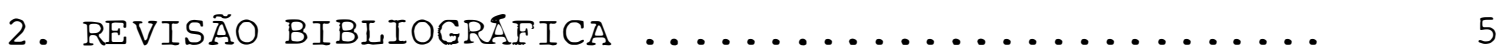

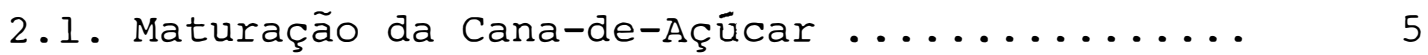

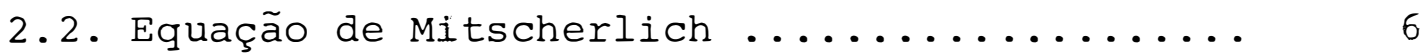

2.3. Método de Marquardt para a Estimação de Parâ metros de Modelos não-lineares ............ 9

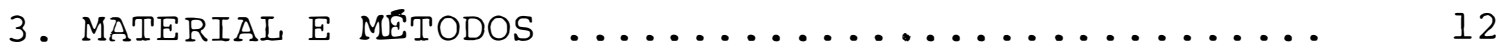

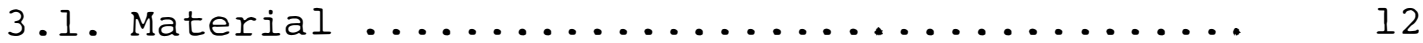

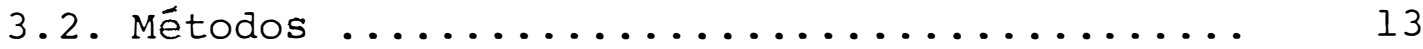

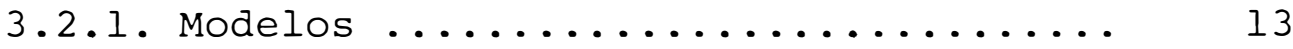

3.2.1.1. Estimativa dos Parâmetros .. 14

3.2.1.2. Aplicação do Método ao Mode$10 \ldots \ldots \ldots \ldots \ldots \ldots \ldots \ldots$

3.2.1.3. Análise de Variância ...... 28

3.2.1.4. Cálculo do máximo das funçoes 32

3.3. Programação da metodologia ............ 34

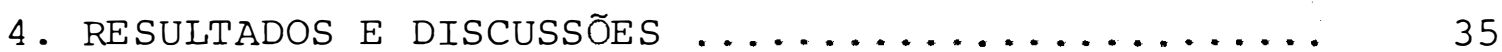

4.1. Estudo dos modelos .................. 35

4.1.1. Segunda aproximação de Mitscherlich . $\quad 35$

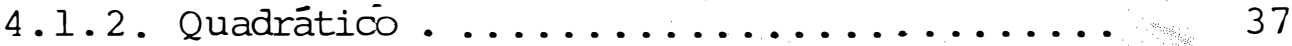

4.2. Valores iniciais da estimativa dosparâmetros do modelo $\mathrm{Y}=\mathrm{A}\left[1-10^{-\mathrm{C}(\mathrm{X}+\mathrm{b})}\right] \cdot 10^{-\mathrm{K}}(\mathrm{x}+\mathrm{b})^{2} \ldots \ldots 37$

4.3. Ajustamento aos modelos ............... 38 
Pāgina

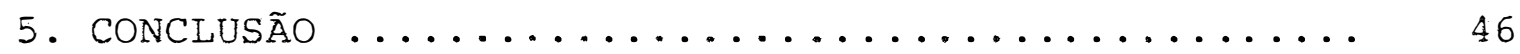

6. BIBLIOGRAFIA $\ldots \ldots \ldots \ldots \ldots \ldots \ldots \ldots \ldots \ldots \ldots \ldots$

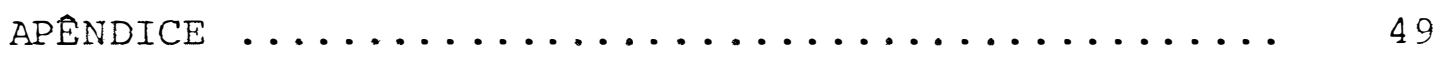


vii.

\section{ESTUDO DE FUNÇÕES CUE SE AJUSTAM A MATURAÇÃo \\ DA CANA-DE-AÇOCAR}

Autora: Margareth C. Toyama vdo

Orientador: Roberto Simionato Moraes

RESUMO

A fim de verificar a melhor equação que adapta se ao fenômeno da maturação da cana-de-açūcar, permitindo uma melhor análise do aproveitamento industrial, foram estudados três modelos, que são:

i) Segunda Aproximação de Mitscherlich:

$$
Y=A\left[1-10^{-C(x+b)}\right] \cdot 10^{-k(x+b)^{2}}+e ;
$$

por que a porcentagem em peso de sacarose da cana-de-açúcartem um crescimento rápido e um decréscimo mais lento.

\section{ii) Suadrātico:}

$$
Y=A+B X+C X^{2}+e ;
$$

por ser utilizado pelos técnicos atualmente. 


\section{iii) Raiz Suadrada$$
Y=A+B+C \sqrt{X}+e,
$$

por que esta equação segue aproximadamente os dois modelos anteriores.

Para estudar os três modelos foram utilizados os resultados analíticos de pol\% cana, ou seja, a porcentagem em peso de sacarose da cana-de-açūcar, obtidos pela Usina São João, Araras (S.P.).

Inicialmente, fez-se a análise de variância em parcelas subdivididas e como a interação época $x$ variedade ( $\mathrm{E} \times \mathrm{V}$ ) foi altamente significativa estudou-se os desdobramentos da época dentro das variedades ( $E \mathrm{~d} . \mathrm{V}_{\mathrm{i}}$ ) e em seguida o efeito da regressão nos desdobramentos.

As estimativas dos parâmetros do modelo (i) foram feitas pelo método de MARৎUARDT (1963); para (ii) e (iii) foram feitas pelométodo dos mínimos quadrados comum, através do pro grama biblioteca, do Centro de Processamento de Dados da ESALS!.

Em seguida fêz-se a análise de regressão e tam bém o gráfico para cada variedade para os três modelos e concluiu-se que:

- o modelo (ii) foi o que melhor se äaptou ao fenômeno de maturação como pode ser visto pelos valores do coe ficiente de determinação $\left(R^{2}\right)$, bem como visualmente pelo pró- 
prio gráfico. Mas, neste modelo não é indicada a extrapolação.

- O modelo (iii) foi o que pior se comportou, não sendo portanto aconselhável a sua utilização.

- O modelo (i) teve uma adaptação pouco inferior ao quadrático, porém os problemas relativos a extrapolação são menores. 


\title{
A STUDY OF FUNCTION SITTING \\ SUGAR CANE RIPENING
}

\author{
Author: Margareth C. Toyama Udo \\ Adviser: Roberto Simionato Moraes
}

\section{SUMMARY}

The main purpose of this dissertation is to verify the best equation relating sugar cane ripening and time, yielding a better analysis of industrial exploitation. Three models are attacked

(i) Second Mitercherlich Approximation:

$$
Y=A\left[1-10^{-C(x+b)}\right] \cdot 10^{-k(x+b)^{2}}+e
$$

$$
\begin{aligned}
& \text { (ii) Suadratic Model } \\
& Y=A+B x+C x^{2}+e \\
& \text { (iii) Square Root Model } \\
& Y=A+B X+c \sqrt{X}+e
\end{aligned}
$$

Analitic results of percentage of saccharose weight are used to study the three models, obtained by são João Sugar Mill, Araras (S.P.) . 
Initially, an analysis of variance is obtained from a split-plot design. By virtue of significance of variety $x$ epoch component a further natural decomposition is done of epoch within varieties followed by linear regression of epochs within varieties.

Parameters estimates of model (i) is obtained by MARSUARDT (1963); for models (ii) and (iii) usual least squares method is applied.

For each model and each variety, data fitting is presented reaching the following main conclusions:

- Model (ii) is the best among the three model fitting when sugar-cane ripening is considered, conclusion based on $\mathrm{R}^{2}$ criterion, but extrapolation is not recommended.

- Model (iiil is the worst, being your utilization not recommended.

- Model (i) is not the best but avoids extrapolation problems. 


\section{INTRODUÇÃO}

A fim de obter o melhor rendimento industrial de açūcar e álcool da cana-de-açúcar é necessário conhecer o seu estágio de maturação. Este estāgio é verificado pelos teores de sacarose e açücares redutores que se apresentam no decorrer do período de safra.

Porém, è necessário saber qual o comportamento varietal em relação ao teor de sacarose durante o período de aproveitamento industrial. Para todas as variedades este período, inicia-se quando o teor de sacarose está ao redor de 13\%. Com base na duração do período de aproveitamento industrial, as variedades podem ser classificadas em longos, médios e curtos.

Variedades precoces são as que apresentam um teor mínimo de sacarose para a moagem no início da safra.

Variedades médias são as que se apresentam em éstāgio de maturação para a. moagem no meio da safra. 
Variedades tardias são aquelas que podem ser industrializadas no final da safra.

Entretanto, há uma tendência para a substituição dos conceitos de precoce, média e tardia por rica, média e pobre em sacarose, respectivamente. Isto porque em qualquer época da safra, uma variedade precoce apresenta teores de sacarose mais elevados do que as variedades médias e tardias, e as médias, por sua vez, são mais ricas do que as tardias.

Toda curva de maturação de uma determinada variedade de cana-de-açūcar tende a mudar de uma safra a outra, mas manterá sempre um estágio idêntico dentro dos conceitos citados anteriormente.

o principal objetivo deste trabalho é a determinação das equações das curvas de maturação para verificar qual delas melhor adapta-se ao fenômeno, permitindo uma melhor análise do aproveitamento industrial destas variedades. Este aumento na eficiência da agro-indústria é de grande importância para - país seja na produção de açūcar, seja na de álcool. com este objetivo serão estudados três modelos, procurando-se deter minar qual deles melhor ajusta-se ao fenômeno que são:

\subsection{Segunda Aproximação de Mitscherlich}

Neste modelo a porcentagem em peso de sacarose da cana-de-açūcar (Pol\% cana) tem crescimento rápido seguido de um decréscimo mais lento. Ou seja, 


$$
Y=A\left[1-10^{-c(x+b)}\right] \cdot 10^{-k(x+b)^{2}}+e ;
$$

onde $\mathrm{Y}$ é o valor de Polo cana, $\mathrm{x}$ os meses, $\mathrm{k}$ (conhecido como fator de prejuízol, ce b são os parâmetros. Como o modelo é não-linear, os parâmetros serão estimados pelo método de MARQUARDT (1963), que consiste na combinação dos métodos de linearização e "steepest-descendent".

\subsection{Quadrático}

Este modelo é utilizado atualmente pelos técnicos. Nele a porcentagem em peso de sacarose de cana-de-açúcar tem crescimento e decréscimo iguais em torno do seu ponto de māximo. ou seja,

$$
\mathrm{Y}=\mathrm{A}+\mathrm{BX}+\mathrm{Cx}^{2}+\mathrm{e}
$$

onde $\mathrm{Y}$ é o valor de Pol\% cana, $\mathrm{x}$ os meses e A,B,C são os parâmetros.

\subsection{Raíz Quadrada}

Este modelo segue, aproximadamente os dois modelos anteriores. E dada por:

$$
Y=A+B X+C \sqrt{x}+e
$$


onde $Y$ é o valor de Polo cana, $\mathrm{X}$ os meses e A, B, C são os pạ ràmetros. 


\section{REVISÃO BIBLIOGRÃFICA}

\subsection{Maturação da Cana-de-açūcar}

BRIEGER (1964), afirma que o melhor método para a determinação do estágio de maturação da cana - de - açūcar consiste na análise laboratorial do caldo.

O autor afirma ainda que para pequenas indústrias, fornecedores de cana-de-açúcar e organizações que não dispõem de laboratório, a determinação do teor de sacarose pode ser feita utilizando-se o refratômetro de campo, efetuando-se a leitura do Brix Refratométrico. A refração da luz através desse caldo determinará a quantidade de sólidos existentes, cujo valor é aproximadamente igual ao teor de sacarose. O mínimo recomendado na leitura do refratômetro é 18 \& Brix, para aconselhar o corte do canavial.

BRIEGER (1965), apresentou os resultados de três anos de estudos, com quinze variedades de cana-de-açúcar, 
do teor de sacarose determinados quinzenalmente de abril à no vembro, também afirma que as variedades reagem diferentemente ao fator clima com relação ao amadurecimento permitindo classificá-las em precoces, médias e tardias.

o autor chamou de Período Otil de Industrialização (P.U.I.) o compreendido entre os teores de sacarose na cana de $12 \%$ a 16\%. Recomendou escolher as variedades em função do P.U.I., sendo preferível os precoces de P:U.I. longo, pois a indústria te 'rá possibilidade de moer a cana dessas variedades desde o inícioaté o fim da safra.

MIOQUE (1968) afirma que o fator mais importan te para se obter alto rendimento industrial é a utilização de matéria prima rica em sacarose; logo, o período de safra deve coincidir com a época da maturação mais completa da cana-deaçucar.

O autor apresenta também o diagrama do P.U.I. de diversas variedades da cana-de-açúcar

2.2. Equação de Mitscherlich

Segundo SILVA (1978), Mitscherlich apresentou, em 1909 o modelo:

$$
Y=A\left[1-10^{-C(x+b)}\right] \text {, }
$$

onde $\mathrm{x}$ representa a dose de nutriente $e \mathrm{Y}$ a produção. 
Nesta equação quando as doses de nutriente crescem, cresce tam bēm a produção.

Segundo SILVA (1978), Mitscherlich, apresentou em 1928 a segunda aproximação:

$$
Y=A\left[1-10^{-C(x+b)}\right] \cdot 10^{-k(x+b)^{2}}
$$

onde $k$ é o fator de prejuízo. O autor desenvolveu este modelo já que a equação (2.2.1.) não explica o fato da produção decrescer para doses de nutrientes excessivamente altas.

A partir da equação diferencial:

$$
\frac{1}{y} \frac{d y}{d x}=c_{1} \frac{10^{-c x}}{1-10^{-c x}}-2 k x
$$

chega-se a equação (2.2.2.).

PIMENTEL GOMES E MALAVOLTA (1949) demonstraram a primeira e a segunda aproximação de Mitscherlich.

A partir da equação diferencial:

$$
\frac{d y}{d x}=k(A-y)
$$

onde y é a produção da planta, $x$ a quantidade de fertilizante assimilável à sua disposição, A e k são constantes positivas, demonstrara a primeira aproximação, dada por (2.2.1.). Esta equa ção sữãe sempre uma produção mäxima inatingível. 
Em seguida, apresentaram a partir da equação diferencial:

$$
\frac{1}{y} \frac{d y}{d x}=c_{1} \cdot \frac{10^{-c x}}{1-10^{-c x}}-2 \mathrm{kx}
$$

como chega-se a segunda aproximação de Mitscherlich.

Os autores afirmaram que os dados de adubação adaptaram-se melhor a segunda aproximação de Mitscherlich. Mas ela apresenta o inconveniente de ter um parâmetro a mais. Além disso, o efeito do fator $10^{-k(x+b)^{2}}$ só inicia-se para valores relativamente grandes de $x$, isto é, para adubações excessivamente pesadas. Portanto, excluindo-se os casos extremos é sempre preferível utilizar-se de (2.2.1.). A partir des sa conclusão, os autores, passaram a estudar apenas a equação (2.2.1.) e apresentaram o cálculo dos trēs parâmetros: A, c e b.

Segundo SILVA (1978), Mitscherlich apresentou em 1954 a importância da segunda aproximação, equação (2.2.2), pois através dela pode-se efetuar um estudo mais realista. Nes sa aproximação pode-se determinar quando o efeito do adubo torna-se prejudicial à planta.

o autor mostrou que a curva resposta nesse caso atinge o máximo passando daí a decrescer de maneira muito lenta, sem apresentar nenhuma maneira de se estimar os parâmetros. 
PIMENTEL GOMES A NOGUEIRA (1975) mostraram a partir do exemplo de regressão quadrática, o perigo da extrapolação, pois esta pode conduzir a absurdos. Isto se verifica, pois após certo período a curva de produção cai rapidamente; o que não ocorre na realidade. Por isso, os autores recomendaram a primeira aproximação de Mitscherlich, para este caso, já que até certo ponto, sua curva resposta permite extrapolação.

$$
\begin{aligned}
& \text { SILVA (1978) estudou três modelos: } \\
& \text { i) } Y=A\left(1-10^{-C(x+b)}\right)+e ; \\
& \text { ii) } Y=A\left(1-10^{-C(x+b)}\right) 10^{-k(x+b)^{2}+e ;} \\
& \text { iii) } Y=A+B x+C x^{2}+e .
\end{aligned}
$$

aplicados à dados de adubação de trigo.

A autora concluiu que a equação (ii) foi a que melhor se ajustou, principalmente quando há aplicação de altas doses de fertilizantes o que acarreta uma queda na produção.

2.3. Método de MARQUARDT para a Estimação de Parâmetros de Modelos não-lineares.

MARQUARDT (1963) introduziu um novo método para a estimação de parâmetros, para modelos não-lineares. o mé 
todo é uma conciliação entre "steepest-descendent" e o de linearização.

Vários autores têm utilizado este método dentre eles SALVIANO (1973) apresentou o modelo não-linear:

$$
y_{t}=\alpha \cdot \beta^{x_{t}}+E_{t} ; t=1,2, \ldots, n \text {. }
$$

onde $\beta=1+i$, sendo $i$ uma constante desconhecida, $x$ é um valor fixo; y é a variável aleatória, e $\alpha, \beta$ são os parâmetros a serem estimados e $E_{t}$ é o erro aleatórío do modelo. Para a solução do problema o autor estudou três métodos:

i) de Gauss-Newton,

ii) do Gradiente e

iii) de Marquardt.

Desses processos o autor afirmou ser o terceiro o melhor mëtodo, pois elimina as desvantagens dos dois outros, isto é, sempre alcança a estimação desejada e usa um número mínimo de iterações. BARD (1974) estudou os métodos de Newton, GaussNewton e Marquardt. Apresentou todo o desenvolvimento teórico dos mesmos e um exemplo numérico. O autor afirmou ser o último método de grande utilidade prática. SILVA (1980) estudou três métodos de estimação de parâmetros do modelo:

$$
Y=A\left[1-10^{-C x}\right] \cdot 10^{-k x^{2}}+e
$$


aplicados a dados de maturação da cana-de-açūcar cujos métodos são Newton, modificados de Gauss-Newton e Marquardt. A autora concluiu que o último método é mais apropriado para o modelo, pois conduz a uma soma de quadrados dos desvios de residuos meno res que os demais. Além disso, na maioria dos casos este método apresentou um nümero menor de iterações.

Portanto, como o método conduz a bons resultados será feita a utilização do mesmo. 


\section{MATERIAL E METODOS}

\subsection{Material}

Os dados foram gentilmente cedidos pela Cooperativa Central dos Produtores de Açūcar e Alcool do Estado de São Paulo (COPERSUCAR). Estes dados são resultados analíticos de Polo cana, que é a porcentagem em peso de sacarose, de terminada através de métodos apropriados como pode ser visto em publicação da C.T.C. - COPERSUCAR (1980).

Na tabela 1l, do apêndice, são apresentados os resultados analiticos de Polo cana em ensaios de competição de variedades de cana-de-açūcar. Estes experimentos foram conduzidos na Usina São João, Araras (S.P.), no ano agrícola de 1979 com cana-planta das variedades CB40-13, CB41-76, CB53-98, CB47-355, CP51-22, IAC48/65, IAC52/150 e Na56-79. Para cada variedade são dados três repetiçães de Polo cana, onde cada repetição é una análise tecnológica de uma amostra de quinze colmos escolhidos ao acaso, por toda a área. Cada uma dessas amostras foram realizadas no mesmo dia ou no máximo vinte e quatro horas após a coleta, 
pois caso contrário, os resultados analitìcos poderiam estar totalmente comprometidos.

\subsection{Métodos}

\subsubsection{Modelos}

Os modelos a serem testados são:

$(3.2 .1 . a) Y_{i}=A\left[1-10^{-c\left(x_{i}+b\right)}\right] 10^{-k\left(x_{i}+b\right)^{2}}+e_{i}$

onde: $\dot{Y}_{i}$ é a porcentagem de sacarose contida na cana-da-açúcar (Pol\% cana)

$x_{i}$ é o mês onde se determina o Pol\% cana

$A, b, c$ e $k$ são os parâmetros da função.

$e_{i}$ é o erro aleatōrio, onde $e_{i} \cap N\left(0, \sigma^{2}\right)$

$(3.2 .1 . b) \mathrm{Y}_{i}=\mathrm{A}+\mathrm{Bx_{i }}+\mathrm{Cx_{i } ^ { 2 }}+\mathrm{e}_{i}$

onde: $Y_{i}$ e a porcentagem desacarose contida na cana-de-açúcar (Pol\% cana).

$x_{i}$ é o mês onde se determina o Polo cana.

A, B, C são os parâmetros da função

$e_{i}$ é o erro aleatōrio, onde $e_{i} \cap N\left(0, \sigma^{2}\right)$.

(3.2.1.c) $Y_{i}=A+B x_{i}+C \sqrt{x_{i}}+e_{i}$. 
onde: $Y_{i}$ é a porcentagem de sacarose contida na cana-de-açú $\operatorname{car}$ (Pol\% cana).

$x_{i}$ é $\circ$ mês onde se determina o Polo cana.

A, B e C são os parâmetros da função.

$e_{i}$ é o erro aleatório, onde $e_{i} \cap \mathrm{N}\left(0, \sigma^{2}\right)$

3.2.1.1. Estimativa dos Parâmetros

3.2.1.1.1. Método para o ajuste de funções lineares nos parâmetros pelo método dos minimos quadrados

Para o cálculo da estimativa dos parâmetros, de funções lineares nos parâmetros, aplica-se o método dos mínimos quadrados, aos modelos 3.2.1.b e 3.2.1.c.

Dados $n$ pares de valores $\left(x_{i}, y_{i}\right) ; i=1,2, \ldots, n$, tem-se gue $x_{i}$ representa os meses, enquanto $Y_{i}$ é a quantidade de Pol o cana, no respectivo mês. Então, a equação na forma geral será:

$$
Y=X \beta+\varepsilon
$$

onde:

$$
x=\left(\begin{array}{ccc}
1 & x_{1} & x_{1}^{2} \\
1 & x_{2} & x_{2}^{2} \\
\vdots & \vdots & \vdots \\
\vdots & \vdots & \vdots \\
1 & x_{n} & x_{n}^{2}
\end{array}\right) \quad \text { quadrático e }
$$




$$
x=\left(\begin{array}{ccc}
1 & x_{1} & \sqrt{x_{1}} \\
1 & x_{2} & \sqrt{x_{2}} \\
\vdots & \vdots & \vdots \\
1 & \vdots & \vdots \\
1 & x_{n} & \sqrt{x_{n}}
\end{array}\right) \quad \text { raiz quadrada, }
$$

$\beta$ é o vetor dos parâmetros, $\varepsilon$ o vetor de erros e Y è o vetor das quantidades analiticas de Pol o cana.

Aplicando.o método dos mínimos quadrados temse o sistema de equações normais cuja solução é:

$$
\bar{B}=\left(X^{\prime} X\right)^{-1} X^{\prime} Y,
$$

que fornece a estimativa dos parâmetros.

Para o modelo 3.2.1.a os parâmetros serão estimados pelo método de Marquardt, apresentado a seguir.

3.2.1.1.2. Método de Marquardt para o ajuste de funções de regressão não linear

Este método foi desenvolvido por MARQUARDT (1963), e através dele serão estimados os parâmetros do mode$103.2 .1 . a$.

Os vários métodos de estimativa dos parâmetros não-lineares por mínimos quadrados são baseados em duas apro- 
ximações. Por um lado o método de linearização e por outro o do "steepest-descendent".

O método de Marquardt representa uma ótima interpolação entre os métodos de linearização e "steepest-descendent".

\section{Exposição do modelo}

Seja o modelo:

$$
\begin{aligned}
y_{i} & =f\left(x_{1}, x_{2}, \ldots, x_{m} ; \beta_{1}, \beta_{2}, \ldots, \beta_{k}\right)+e_{i} \\
& =f(x, \beta)+E,
\end{aligned}
$$

para $\quad i=1,2, \ldots, n ;$ onde:

$\mathrm{x}_{\text {ik }}$ são as variáveis independentes, $\mathrm{k}=1,2, \ldots, \mathrm{m}$

$B_{i}$ são valores populacionais de k-parâmetros e

$e_{i}$ são os erros aleatórios independentes, ou de $e_{i} \cap N\left(0, \sigma^{2}\right)$, para todo i.

Indicam-se os pontos dados por:

$$
\left(Y_{i} ; x_{1 i} ; x_{2 i}, \ldots, x_{m i}\right) \text {, para } i=1,2, \ldots, n,
$$

cujo problema é minimizar a função $\phi(B)$ dada por:

$$
\phi(\beta)=\sum_{i=1}^{n}\left[Y_{i}-f\left(x_{i k^{\prime}}, \beta\right)\right]^{2}
$$

\section{Método}

Inicialmente toma-se o desenvolvimento da fun- 
Ção $f(x, B)$ pela série de Taylor através de termos lineares no ponto $\beta=\beta_{0}$, onde $\beta_{0}$ é o ponto inicial. Daí tem-se:

$$
f(x, \beta)=f\left(x, \beta_{0}\right)+\sum_{j=1}^{k}\left(\frac{\partial f_{i}}{\partial \beta_{j}}\right)\left(d_{t}\right)_{j} \quad \text { (3.2.1.1.2.c) }
$$

onde $\left(a_{t}\right)_{j}=\beta_{j}-\beta_{0}$

Substituindo-se, agora, este resultado

em

(3.2.1.1.2.b) tem-se:

$$
\phi(\beta)=\sum_{i=1}^{n}\left(y_{i}-f\left(x, \beta_{0}\right)-\sum_{j=1}^{k} \frac{\partial f_{i}}{\partial \beta_{j}}\left(a_{t}\right)_{j}\right)^{2}
$$

Agora $\left(d_{t}\right)_{j}$ aparece linearizado. Pode-se assim empregar o método dos mínimos quadrados fazendo

$$
\frac{\partial \phi(\beta)}{\partial \beta_{j}}=0, \text { para todo } j
$$

Então, determina-se $d_{t}$ resolvendo:

$$
A a_{t}=-q
$$

onde:

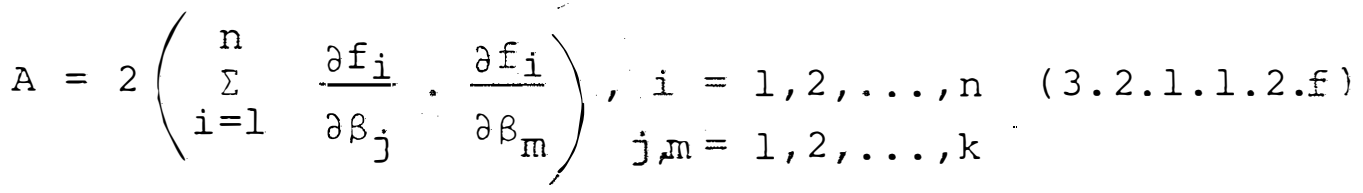

$$
\begin{aligned}
& q=2\left(\sum_{i=1}^{n}\left(Y_{i}-f_{i}\right) \frac{\partial f_{i}}{\partial \beta_{j}}\right) ; \begin{array}{l}
i=1,2, \ldots, n \quad(3.2 .1 .1 .2 . g) \\
j=1,2, \ldots, k
\end{array}
\end{aligned}
$$


Na prática, isto equivale a encontrar o valor correto de $\beta$ por somente uma fração $d_{t}$ lt é usado para desig nar que d foi calculado usando a série de Taylor].

O importante é obter a direção $d_{t}$ do próximo passo. O parâmetro deve ser corrigido por uma fração $d_{t}$ compatível, pois pode ocorrer um passo inaceitável, fazendo com que saia da região onde $f(x, \beta)$ pode adequadamente ser representada por (3.2.1.1.2.c) causando assim a divergência do processo. Vários métodos tomam o tamanho do passo como $\rho \cdot a_{t}, \quad 0 \leqslant \rho \leqslant 1 ; j a ́$ que a direção é específicado por $d_{t}$. Assim a divergência raramente ocorre.

O método "steepest-descendent" por contraste simplesmente, toma a direção do gradiente negativo de $\phi(B)$, assim:

$$
\mathrm{d}_{g}=-\left(\frac{\partial \phi}{\partial \beta_{1}}, \frac{\partial \phi}{\partial \beta_{2}}, \ldots, \frac{\partial \phi}{\partial \beta_{k}}\right)^{\prime} ;
$$

onde: ' indica a matriz transposta.

Tanto no método de Iinearilização como no "stee pest-descendent" é necessário o controle cuidadoso do tamanho do passo. Ainda assim, a convergência é lenta em muitos casos.

No algoritmo a ser descrito, a direção e o tamanho do passo são determinados simultaneamente. 
Base Teórica do Algorítmo de Determinação de Direção e Tamanho do Passo

A base teórica do algorítmo está contida em vấ rios teoremas, sendo que os teoremas 1 e 2 são devidos a Morrison e citados por MARQUARDT (1963), onde são apresentadas as äemonstrações.

Teorema 1: Seja $\lambda \geqslant 0$ arbitrário e do satisfazen do a equação:

$$
(A+\lambda I) d_{0}=-q
$$

Então do $_{0}$ minimiza $\phi(X, \beta)$ na esfera cujo raio \|d \| satisfaz,

$$
\|\mathrm{a}\|^{2}=\left\|\mathrm{d}_{0}\right\|^{2}
$$

onde \| $\|$ è a indicação de norma.

Teorema 2: Seja d $(\lambda)$ a solução de (3.2.1.1.2.h) para um dado valor de $\lambda$. Então $\|d(\lambda)\|^{2}$ é uma função decrescente continua de $\lambda$, tal que quando $\lambda \rightarrow \infty,\|d(\lambda)\|^{2} \rightarrow 0$.

Teorema 3: Seja $\gamma \circ$ ângulo entre $d_{0}$ e $d_{g}$. Então $\gamma$ é uma função monótona decrescente e contínua de $\lambda$, tal que, quando $\lambda \rightarrow \infty, \gamma \rightarrow 0$. Desde que $\mathrm{d}_{\mathrm{g}} \overline{\mathrm{e}}$ independente de $\lambda$, isto segue que do sofre uma rotação na direção de $d_{g}$, com $\lambda \rightarrow \infty$. 


\section{Construção do Algoritmo}

A solução, $d_{t}$, de (3.2.1.1.2.e) tem a propriedade de ser invariante por transformações lineares no espaço dos parâmetros $\beta$, o que não ocorre com o método "steepest-des cendent". Então é necessário criar uma escala para o espaço dos parâmetros $\beta$. De maneira conveniente MARQUARDT (1963) sugere a seguinte escala,

$$
\left(A+\lambda B^{2}\right) d=-q
$$

onde a matriz B é definida como:

$$
B=\left(b_{i i}\right)=\left\{\begin{array}{ll}
\left(a_{i i}\right)^{1 / 2}, & \text { se } a_{i i} \neq 0 \\
1, & \text { se } a_{i i}=0
\end{array} \quad(3 \cdot 2 \cdot 1 \cdot 1 \cdot 2 \cdot j)\right.
$$

ao invés de se tomar o sistema dado em (3.2.1.1.2.e).

Portanto, $\mathrm{B}^{2}$ é uma matriz diagnonal cujos elementos coincidem com o valor absoluto dos elementos da diagonal de A. Em seguida, pré-multicando-se o sistema (3.2.1.1.2.i) por $\left(A+\lambda B^{2}\right)^{-1}$ tem-se:

$$
a=-\left(A+\lambda B^{2}\right)^{-1} \cdot q \quad(3.2 .1 .1 .2 .1)
$$

que é a direção aceitável, pois $\left(A+\lambda B^{2}\right)^{-1}$ è uma matriz defi nida positiva.

Algorítmo do Método para seleção de $\lambda_{i}$

1) Para $i=1$, inicia-se $\operatorname{com} \lambda=10^{-2}$.

2) para a i-ésima iteração. Calculam-se: 


$$
\begin{aligned}
d & =-\left(\dot{A}_{i}+\lambda B_{i}^{2}\right)^{-1} \cdot q_{i}, \\
\beta^{(I)} & =\beta_{i}+d, e \\
\phi^{(I)} & =\phi\left(\beta^{(I)}\right)
\end{aligned}
$$

3) se $\phi^{(1)}<\phi_{i}$, aceita-se $\beta_{i-1}=\beta^{(1)}$ e troca-se $\lambda$ pelo máx $(0,1 . \lambda ; \varepsilon)$ onde considera-se $\varepsilon$ como sendo igual a $10^{-7}$.

4) se $\left(d^{\prime} \cdot q_{i}\right)^{2} /\left[\left(q^{\prime} i \cdot q_{i}\right)\left(d^{\prime} \cdot d\right)\right]<1 / 2$, troca-

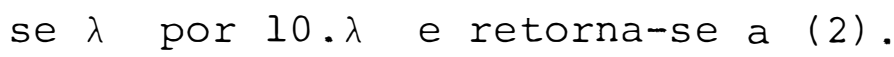

o presente algorítmo pode requerer cálculo de d para vários valores de $\lambda$ em uma simples iteração. Isto po de ser evitado trocando-se (4) por:

4a) Encontrar umvalor $\rho_{i}$ suficientemente pequeno tal que:

$$
\phi\left(B_{i}+\rho_{i} \cdot d\right)<\phi_{i}
$$$$
\text { Aceita-se } \beta_{i+1}=\beta_{i}+\rho_{i} \cdot d \text { e troca-se } \lambda \text { por }
$$

10. $\lambda$.

Segundo BARD (1974) o melhor valor de $p_{i}$ é obtido tomando-se o minimo de:

$$
a+b \cdot \rho_{0}+c \cdot \rho_{0}^{2}=\alpha,
$$


onde: $\quad \mathrm{a}=\phi\left(\beta_{i}\right)$

$$
\begin{aligned}
& \mathrm{b}=-q^{\prime}{ }_{i} \cdot A^{-1} \cdot q_{i}=q^{\prime}{ }_{i} \cdot d_{i} \\
& \alpha=\phi\left(\beta_{i}+\rho 0 \cdot d_{i}\right) . \\
& c=\left(-q^{\prime}{ }_{i} d_{i} \rho_{0}+\phi\left(\theta_{i}\right)-\phi\left(\theta_{i}+\rho_{0} d_{i}\right) / \rho_{0}^{2}\right.
\end{aligned}
$$

Trata-se de uma função quadrática cujo ponto estacionário é:

$$
\rho=-\frac{b}{2 c}=\frac{-q_{i} a_{i} \rho_{0}^{2}}{2\left[-q^{\prime}{ }_{i} a_{i} \rho_{0}+\phi\left(\theta_{i}\right)-\phi\left(\theta_{i}+\rho_{0} d_{i}\right)\right]}
$$

tomando-se $\rho_{0}$ no intervalo $0<\rho_{0}<t$, onde $t$ é um nümero suficientemente grande.

Com esse algorítmo sempre obtém-se uma vizinhan ça, na qual a série de Taylor dá uma adequada representação da função e consequentemente possibilita a determinação do minimo de $\phi(\beta)$.

\section{Fim do Processo Iterativo.}

o processo natural para a finalização é a anulação do gradiente dg. Mas, quase sempre, os cálculos fornecem estimativas dos parâmetros muito próximas do minimo e ainda assim, o gradiente continua com tamanho considerável tornando sua anulação impossível. Por outro lado, cá so haja a divergência, o critério de finalização não funciona, tornando o processo iterativo interminável.

Logo, um critério prático é finalizar o ciclo iterativo quando as iterações suplementares falharem na mudan 
ça significativa dos parâmetros. Isto é, dado um conjunto $\varepsilon_{j}(j=1,2, \ldots, k)$ aceita-se $\beta_{i+1, j}$ como a solução $\beta^{*}$, se:

$$
\left|\beta_{i+1, j}-\beta_{i, j}\right|<\varepsilon_{j}
$$

onde $\beta_{i, j}$ é o j-ésimo componente de $\beta_{i}$.

$$
\text { Segundo MARQUARDT (1963) os valores } \varepsilon_{j} \text { devem }
$$

ser pré-estabelecidos, e sugere:

$$
\varepsilon_{j}=10^{-2}\left(B_{i, j}+10^{-3}\right)
$$

onde o valor $10^{-3}$ é colocado para evitar os casos em que $\beta_{i, j}$ são próximos de zero.

Terminado o processo iterativo $\operatorname{com} \beta=\beta^{*}$, deve-se saber se $\beta^{*}$ está ou não próximo do mínimo. Assume-se então que se conhece o gradiente $q^{*}=q\left(\beta^{*}\right)$ e a matriz Hessia na $H^{*}=H\left(B^{*}\right)$ (Matriz das derivadas segrmala) ou alguma aproximação sua. Agora, ao seccionar-se a superfície $\phi(\beta)$ ao longo dos eixos $\beta_{j}$, tem-se ura curva cuja equação aproximada nas vizinhanças de $\beta$ * é dada por:

$$
S\left(\beta_{j}\right)=\beta^{*}+\left(q_{j}^{*}\right) \cdot\left(\beta_{j}-\beta_{j}^{*}\right)+\frac{1}{2}\left(\beta_{j}-\beta_{j}^{*}\right) \cdot \cdot H_{j, j}^{*}\left(\beta_{j}-\beta_{j}^{*}\right)
$$

cujo ponto estacionário é:

$$
B_{j}=B_{j}^{*}-q_{j}^{*} / H_{j, j}^{*}
$$

Portanto, o valor $a_{j}=\left|q^{*}{ }_{j} / H^{*}{ }_{j, j}\right|$ é uma medi da de erro na determinação de $\beta$ *. 
Se cada $d_{j}$ é pequeno, na mesma escala na qual $\beta_{j}$ é medido, então $\beta^{\star}$ está bem próximo do mỉnimo de $\phi(\beta)$.

\subsubsection{Aplicação do Método ao Modelo \\ o modelo:}

$$
Y_{i}=A\left[1-10^{-C\left(x_{i}+b\right)}\right] \cdot 10^{-k(x+b)^{2}}
$$

é uma função de uma variável independente $x_{i}$ e de quatro parâa

$$
\text { metros: } \quad \begin{aligned}
\beta_{1} & =\mathrm{A} \\
\beta_{2} & =\mathrm{c} \\
\beta_{3} & =\mathrm{b} \\
\beta_{4} & =\mathrm{k}
\end{aligned}
$$

Derivando, tem-se:

$$
\begin{aligned}
& F_{I}=\frac{\partial Y_{i}}{\partial A}=\left[1-10^{-c\left(x_{i}+b\right)}\right] \cdot 10^{-k\left(x_{i}+b\right)^{2}} \\
& F_{2}=\frac{\partial Y_{i}}{\partial c}=\left(x_{i}+b\right) \cdot A \cdot 10^{-c\left(x_{i}+b\right)} \cdot 10^{-k\left(x_{i}+b\right)^{2}} \cdot L(10) \\
& F_{3}=\frac{\partial Y_{i}}{\partial b}=A \cdot 10^{-k\left(x_{i}+b\right)^{2}} \cdot L(10)\left(\begin{array}{l}
-2 k\left(x_{i}+b\right)+\left(c+2 k\left(x_{i}+b\right)\right) \cdot \\
.10^{-c\left(x_{i}+b\right)}
\end{array}\right) \\
& F_{4}=\frac{\partial Y_{i}}{\partial k}=-\left(x_{i}+b\right)^{2} \cdot A\left[1-10^{-c\left(x_{i}+b\right)}\right] \cdot 10^{-k\left(x_{i}+b\right)^{2}} \cdot L(10)
\end{aligned}
$$




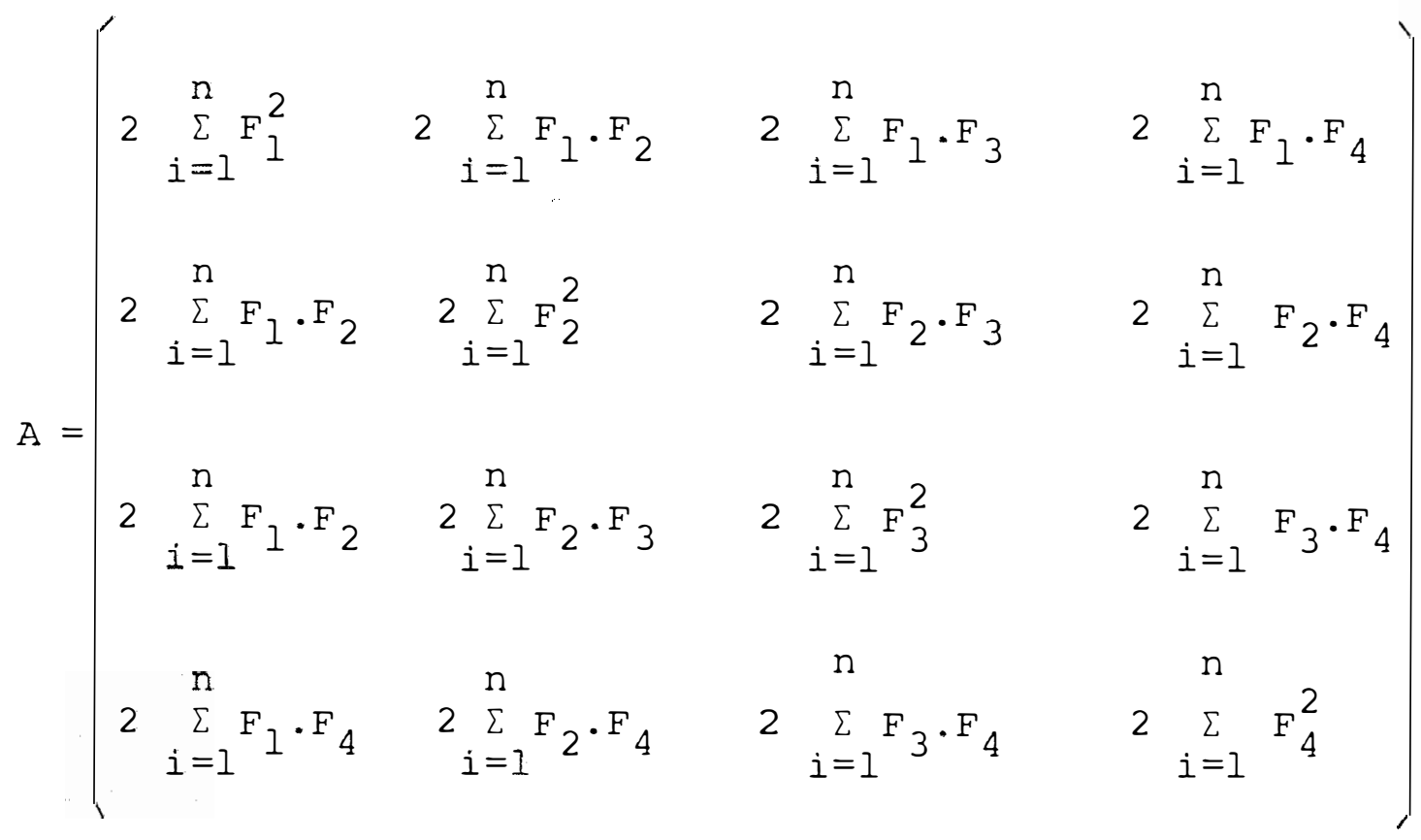

onde:

$$
\begin{aligned}
& F_{1}^{2}=\left[\left(1-10^{-C\left(x_{i}+b\right)}\right) \cdot 10^{-k\left(x_{i}+b\right)^{2}}\right]^{2} \\
& F_{1} \cdot F_{2}=A\left(x_{i}+b\right) \cdot 10^{-C\left(x_{i}+b\right)} \cdot\left[1-10^{-C\left(x_{i}+b\right)}\right] \cdot 10^{-2 k\left(x_{i}+b\right)^{2}} \cdot L(10) \\
& F_{1} \cdot F_{3}=A\left[-2 k\left(x_{i}+b\right)+\left(x+2 k\left(x_{i}+b\right)\right) \cdot 10^{-C\left(x_{i}+b\right)}\right] . \\
& \cdot\left[1-10^{-c\left(x_{i}+b\right)}\right] \cdot 10^{-2 k\left(x_{i}+b\right)^{2}} \cdot I(10) \\
& F_{1} \cdot F_{4}=-A\left(x_{i}+b\right)^{2}\left[1-10^{-c\left(x_{i}+b\right)}\right] \cdot 10^{-k\left(x_{i}+b\right)^{2}} \cdot L(10) \\
& F_{2}^{2}=\left[\left(x_{i}+b\right) \cdot A \cdot 10^{-C\left(x_{i}+b\right)} \cdot 10^{-k\left(x_{i}+b\right)^{2}} \cdot L(10)\right]^{2} \\
& F_{2} \cdot F_{3}=A^{2}\left(x_{i}+b\right) \cdot 10^{-C\left(x_{i}+b\right)}\left(\begin{array}{l}
-2 k\left(x_{i}+b\right)+\left(c+2 k\left(x_{i}+b\right)\right) \\
10^{-c\left(x_{i}+b\right)}
\end{array}\right) \\
& .10^{-2 k\left(x_{i}+b\right)^{2}} \cdot[L(10)]^{2}
\end{aligned}
$$




$$
\begin{aligned}
& F_{2} \cdot F_{4}=-A^{2} \cdot\left(x_{i}+b\right)^{2} \cdot 10^{-c\left(x_{i}+b\right)} \cdot\left[1-10^{-c\left(x_{i}+b\right)}\right] \cdot 10^{-2 k\left(x_{i}+b\right)^{2}} . \\
& \text {. }[L(10)]^{2} \\
& F_{3}^{2}=\left[A \cdot 10^{-k\left(x_{i}+b\right)^{2}} \cdot L(10)\left(-2 k\left(x_{i}+b\right)+\left(c+2 k\left(x_{i}+b\right) \cdot 10^{-C\left(x_{i}+b\right)}\right)\right]^{2}\right. \\
& F_{3} \cdot F_{4}=-A^{2} \cdot\left(x_{i}+b\right)^{2}\left[-2 k\left(x_{i}+b\right)+\left(c+2 k\left(x_{i}+b\right)\right) \cdot 10^{-c\left(x_{i}+b\right)}\right] . \\
& \cdot\left[1-10^{-c\left(x_{i}+b\right)}\right] \cdot 10^{-2 k\left(x_{i}+b\right)^{2}} \cdot[L(10)]^{2} \\
& F_{4}^{2}=\left[-\left(x_{i}+b\right)^{2} \cdot A \cdot\left(I-10^{-c\left(x_{i}+b\right)}\right) \cdot 10^{-k\left(x_{i}+b\right)^{2}} \cdot L(10)\right]^{2}
\end{aligned}
$$

De acordo com (3.2.1.1.2.g) fazendo

$$
\hat{Y}_{i}=A \cdot\left[1-10^{-C\left(x_{i}+b\right)}\right] \cdot 10^{-k\left(x_{i}+b\right)^{2}}
$$

tem-se:

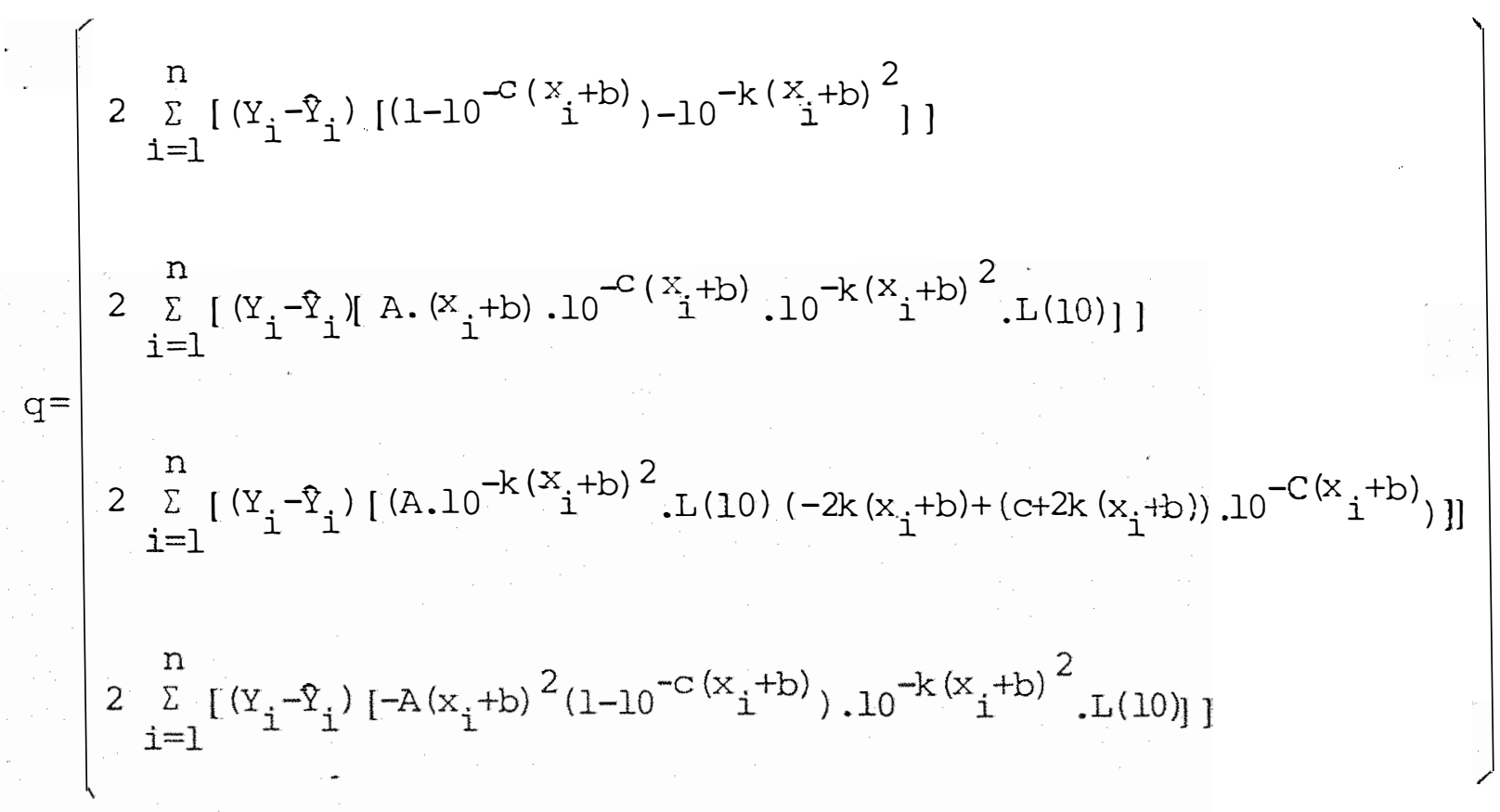

Logo, de acordo com a matriz B em(3.2.1.1.2.j) chega-se ao sistema dado em (3.2.1.1.2.i). 


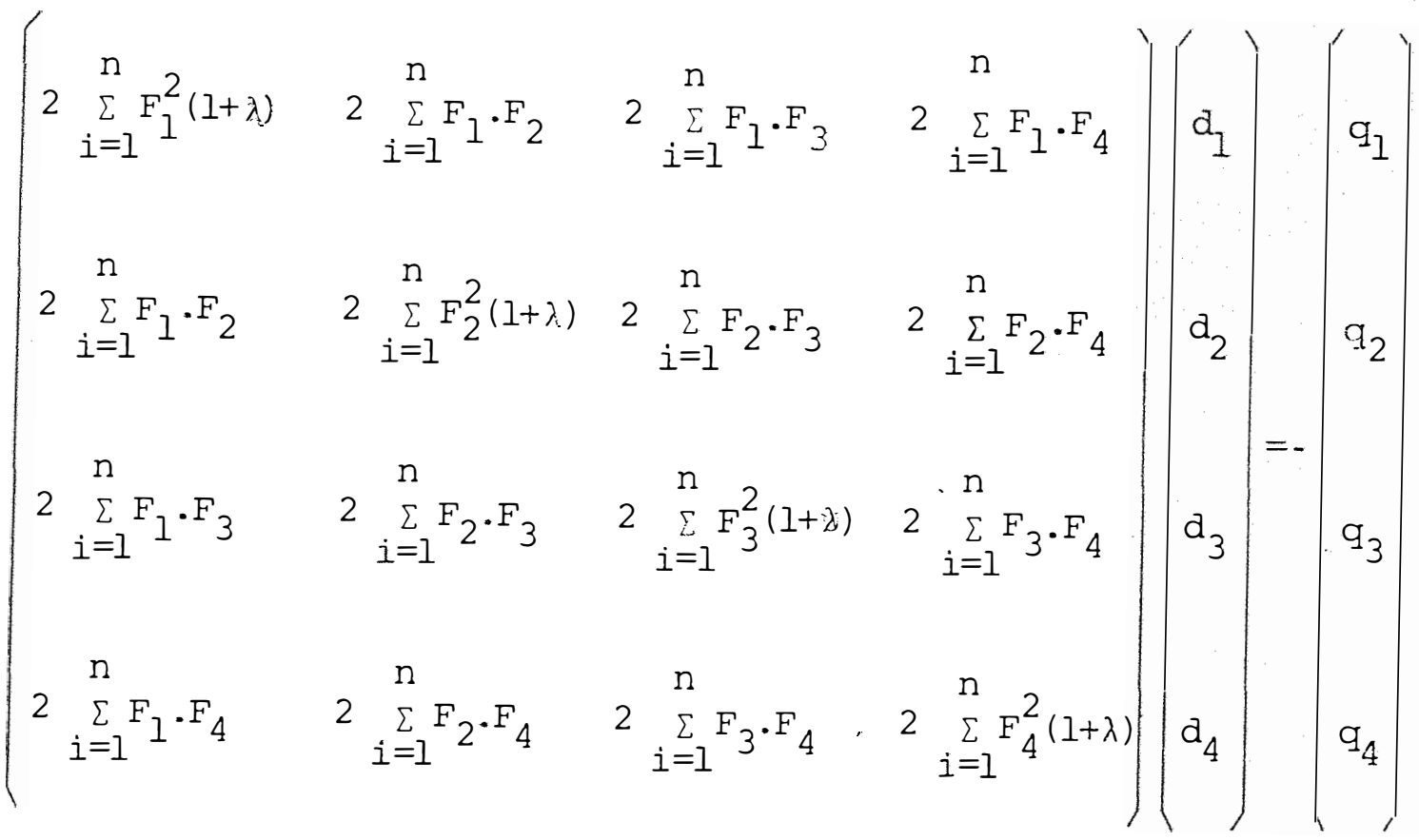

Portanto, de acordo com o valor inicial $\left(\mathrm{A}_{0}, \mathrm{C}_{0^{\prime}}\right.$ $\left.b_{0}, k_{0}\right)$ chega-se a:

$$
\left(A+\lambda B^{2}\right) \cdot\left(\begin{array}{l}
A-A_{0} \\
c-c_{0} \\
b-b_{0} \\
k-k_{0}
\end{array}\right)=-q
$$

A matriz $\left(A+\lambda B^{2}\right)$ é definida positiva nas pro ximidades do mínimo, de modo que escolhido o valor inicial ade quado chega-se ao sistema (3.2.1.1.2.1).

$$
\left(\begin{array}{l}
A-A_{0} \\
c-c_{0} \\
b-b_{0} \\
k-k_{0}
\end{array}\right)=-\left(A+\lambda B^{2}\right)^{-1} \cdot q
$$


Em seguida faz-se um acréscimo aos valores ini

ciais .

$$
\left(\begin{array}{c}
A_{i} \\
c_{i} \\
b_{1} \\
k_{i}
\end{array}\right)=-q\left(A+\lambda B^{2}\right)^{-1}
$$

e continua-se o processo até que o erro seja:

$$
\varepsilon_{\alpha}<10^{-2}\left(\beta_{i, \alpha}+10^{-3}\right),
$$

onde o valor aditivo $10^{-3}$ è colocado para evitar os casos em que $\beta_{i, \alpha}$ são próximos de zero.

3.2.1.3. Análise de Variância

Os ensaios em delineamentos de blocos casualizados em parcelas subdivididas, serão analisados de modousual segundo o esquema apresentado a seguir, na Tabela 1 . 
TABELA 1 - Esquema de Análise de variāncia em parcelas subdivididas.

Causas de variação

Grau de liberdade

Blocos (B)

$b-1$

Variedade (V)

$\mathrm{n}_{\mathrm{a}}-1$

Resíduo (a)

$(b-1)\left(n_{a}-1\right)$

Parcela

$b \cdot n_{a}-1$

Epoca (E)

Interação $\mathrm{ExV}$

Interação $\mathrm{ExB}$

Residuo (b)

$\mathrm{n}_{\mathrm{b}}-1$

$\left(n_{b}-1\right)\left(n_{a}-1\right)$

$\left(n_{b}-1\right)(b-1)$

$\left(n_{b}-1\right)(b-1)\left(n_{a}-1\right)$

Total

$$
n_{a} \cdot n_{b} \cdot b-1
$$

onde: $\quad \mathrm{b}=$ número de blocos

$\mathrm{n}_{\mathrm{a}}=$ número de tratamentos $\mathrm{A}$ nas parcelas, $\mathrm{e}$

$\mathrm{n}_{\mathrm{b}}=$ nümero de tratamentos $\mathrm{B}$ nas sub-parcelas.

Através da análise anterior verifica-se o efei to da interação ExV é significativa, e neste caso, faz-se o desdobramento em época dentro de variedade, como se vê na Tabela 2 . 
TABELA 2 - Esquema de análise de variância com desdobramento da época dentro de variedade.

\begin{tabular}{|c|c|}
\hline Causas de variação & Graus de liberdade \\
\hline Blocos & $b-1$ \\
\hline Variedade & $n_{a}-1$ \\
\hline Resíduo (a) & $(b-1) \quad\left(n_{a}-1\right)$ \\
\hline Parcela & b. $n_{a^{-1}}$ \\
\hline Epoca d. var. i & $\mathrm{n}_{\mathrm{b}}-1$ \\
\hline Epoca d. var. 2 & $\begin{array}{l}\mathrm{n}_{\mathrm{b}}-1 \\
:\end{array}$ \\
\hline $\begin{array}{l}\text { Epoca d. var. } \mathrm{n}_{\mathrm{b}} \\
\text { Residuo (b) }\end{array}$ & $\begin{array}{c}n_{b}-1 \\
\left(n_{b}-1\right)(b-1)\left(n_{a}-1\right)\end{array}$ \\
\hline Total & $\mathrm{n}_{\mathrm{a}} \cdot \mathrm{n}_{\mathrm{b}} \cdot \mathrm{b}-\mathrm{I}$ \\
\hline
\end{tabular}

Obtidos os desdobramentos, caso a época dentro da variedade seja significativa faz-se a análise de variância levando-se em conta a regressão como é apresentada na 'Tabela 3.

TABELA 3 - Esquema da análise de variância levando-se em conta a regressão.

\begin{tabular}{lc}
\hline Causas da variação & Graus de liberdade \\
\hline Regressão & $\mathrm{p}-1$ \\
Desvio de regressão & $\mathrm{n}_{\mathrm{b}}-\mathrm{p}$ \\
Epoca d. var. i & $\mathrm{n}_{\mathrm{b}}-1$ \\
Resíduo (b) & $\left(\mathrm{n}_{\mathrm{b}}-1\right)\left(\mathrm{n}_{\mathrm{a}}-1\right)(\mathrm{b}-1)$
\end{tabular}

onde $\mathrm{p}$ é o número de parâmetros do modelo de regressão. 
Para a segunda aproximação de Mitscherlich a soma dos quadrados dos desvios de regressão serão dados por:

$$
\begin{aligned}
& \text { SQ Desv. }=r \cdot \sum_{i=1}^{n a}\left(\bar{Y}_{i}-\overline{\bar{Y}}_{i}\right)^{2} \\
& \text { E a soma dos quadrados de regressão será dada }
\end{aligned}
$$

por:

SQ Reg. = SQ Época d.Var. i - SQ Desv. Reg.

E, para o modelo quadrático e raiz quadrada a soma dos quadrados de regressão serão dados por

$$
\mathrm{SQ} \operatorname{Reg} \cdot=r\left(\hat{B}^{\prime} X^{\prime} Y-C\right),
$$

e a soma dos quadrados dos desvios de regressão por:

$$
\text { SQ Desv. Reg. = SQ Epoca d. Var. i - SQ Reg. }
$$$$
\text { Quanto ao coeficiente de determinação } R^{2} \text {, se- }
$$
rão calculados por:

$$
\mathrm{R}^{2}=\frac{\mathrm{SQ} \text { Reg. }}{\text { SQ Epoca d. Var. } i}
$$

por que o resíduo não se confunde com os desvios de re gressão. 
3.2.1.4. Cálculo do máximo das funções

3.2.1.4.1. Modelo: segunda aproximação de Mitscherlich

Obtém-se numericamente o máximo da função a par tir do teorema de Rolle.
Algorítmo utilizado no cálculo do máximo da função:

Calcula-se o valor de Pol o cana máximo utilizando-se o computador. Para tanto, verifica-se em que ponto a função muda de crescente para decrescente. Para isso, ini cia-se o processo tomando-se um valor $\Delta x$ arbitrário de distan ciamento de ponto. A partir do valor inicial $\mathrm{x}$ acrescemos a ele $\circ$ valor $\Delta x$, verificando em cada ponto o sinal de $\Delta y$. Quan do há uma mudança de sinal, repete-se o processo tomando - se como $\Delta x \circ$ valor $\Delta x / 2$ e como $x$ inicial $\circ \mathrm{x}$ anterior a mudança de sinal. Repete-se este processo até obter $\left|\hat{Y}_{i+1}-\hat{Y}_{i}\right|<10^{-7}$; isto é, até que a diferença entre o passo atual e o anterior seja menor que $10^{-7}$.

\subsection{Modelo quadrático}

Para este modelo calcula-se o máximo pelo méto do usual, ou seja, fazendo-se a derivada tem-se então:

$$
Y^{\prime}=B+2 C X
$$


Fazendo-se $Y^{\prime}=0$, tem-se o ponto critico, isto é, o mês que a cana-de-açúcar fornecerá a maior porcentagem de sacarose e este mês será:

$$
x=-\frac{B}{2 C}
$$

Agora necessita-se calcular a segunda derivada para se fazer o estudo da concavidade. Neste caso, o objetivo é que esta seja negativa assim a função atingirá o máximo, pois caso contrário teremos um mínimo. Então:

$$
Y^{\prime \prime}=2 C,
$$

logo $Y^{\prime \prime}$ só será negativa quando $C<0$.

Portanto, no mês de maior piodução de sacarose, a produção máxima será:

$$
Y=a-\frac{B^{2}}{4 . C}
$$

3.2.1.4.3. Modelo: raiz quadrada

Analogamente ao modelo (3.2.1.4.2.) tem-se:

$$
Y^{\prime}=B+\frac{C}{2} x^{-1 / 2}
$$

E o ponto crítico, ou seja, o mês de maior pro dução de secarose será: 


$$
x=\frac{C^{2}}{4 \cdot B^{2}}
$$

Calculando-se a segunda derivada tem-se:

$$
Y^{\prime \prime}=-\frac{1}{4} \cdot C \cdot x^{-3 / 2},
$$

substituindo o ponto crítico calculado e acrescentando-se a restrição necessária, chega-se a conclusão que o parâmetro $B$ deve ser negativa. E o máximo da produção será:

$$
Y=A-\frac{C^{2}}{4 B}
$$

\subsection{Programação da metodologia}

Para atingir os objetivos propostos fez-se as programações do método de MARQUARDT (1963) para estimar os pa râmetros e o cálculo do máximo da função para o modelo. Segun do aproximação de Mitscherlich em linguagem FORTRAN IV; para os outros modelos utilizou-se o programa biblioteca, do Centro de Processamento de Dados, do Departamento de Matemática e Estatistica da ESALQ ondecom O auxílio do computador IBM 1130, foram realizados os processamentos.

Os dois programas desenvolvidos estão apresentados no apêndice. 


\section{RESULTADOS E DISCUSSÕES}

\subsection{Estudo dos modelos}

Foram feitos os estudos dos modelos, a fim de verificar os sinais dos parāmetros.

Inicialmente, sabe-se que $Y$ é positivo, nos três casos, pois não há possibilidade de termos um Polo negativo

4.1.1. Segunda aproximação de Mitscherlich

Aplicando log na função obtëm-se:

$$
\begin{aligned}
& \log Y=\log \left[A\left[1-10^{-C(x+b)}\right]\right]+\log 10^{-k(x+b)^{2}} \\
& \log Y=\log A+\log \left[1-10^{-C(x+b)}\right]+\log 10^{-k(x+b)^{2}} \text { (4.1.la) } \\
& \text { Pela restrição de logarítmo, tem-se: } \\
& \text { i) } Y>0
\end{aligned}
$$




$$
\begin{aligned}
& \text { ii) } A>0 \\
& \text { iii) } 1-10^{-C(x+b)}>0 \\
& \text { iv) } 10^{-k(x+b)^{2}>0}
\end{aligned}
$$$$
\text { Da restrição (iii) tem-se: }
$$$$
\frac{10^{c(x+b)}-1}{10^{c(x+b)}}>0, \text { mas } 10^{c(x+b)}>0
$$$$
\text { Logo, } 10^{c(x+b)}>1
$$

Portanto $c>0$ e $(r+b)>0$, devido $x$ ser também sempre positivo.

Da equação (4.1.1.a) tem-se:

$$
k=\frac{1}{(x+b)^{2}}\left(\log A+\log \left(1-10^{-c(x+b)}\right)-\log Y\right)
$$

Como $k>0$, devido a porcentagem de sacarosede cana-de-açúcar tender a decrescer após atingir o máximo e $(\mathrm{x}+\mathrm{b})^{2}>0$, tem-se:

$$
\begin{aligned}
& \log A+\log \left(1-10^{-C(X+b)}\right)-\log Y>0 \\
& \log A>\log \frac{Y}{1-10^{-C(x+b)}} \\
& A>\frac{Y}{1-10^{-C(x+b)}}
\end{aligned}
$$


Pelo item (3.2.1.4.2.) sabe-se que y é um valor positivo; C é negativo e a reta tangente é dada pela fun ção:

$$
Y^{\prime}=B+2 C X
$$

Tem-se também, que o mês de maior percentagem de sacarose, con siderando o fato de.c ser négativo é:

$$
x=\frac{B}{2 C}
$$

Porém, a partir de $\mathrm{x}$ maior que o ponto considerado, isto é, $x>\frac{B}{2}$, a percentagem de sacarose começa a decrescer. Analizando este fato, mais a reta tangente, pode-se ver que quan to maior o valor de C em valor absoluto, maior será a queda da função Y.

Agora, se $x=0$, isto é, um mês antes do cor te, o Pol o cana será o próprio valor de A.

Observação: Quanto ao modelo raiz quadrada não foi estudado, pois ele não apresentou-se adequado para o estudo.

4.2. Valores iniciais da estimativa dos parâmetros do $\underline{\text { modelo }} \cdot \underline{Y=A\left[1-10^{-c}(x+b)\right] \cdot 10^{-k}(x+b)^{2}}$

Geralmente, os modelos não lineares nos parâme 
tros requerem um processo iterativo e para tal é muito importante a seleção de bons valores iniciais. Da escolha destes valores pode depender a convergência ou não do modelo, bem cô mo a rapidez da convergência.

Apesar disso, ainda não existem algoritmos efi cientes para solucionar o problema, sendo quase sempre necessārio recorrer-se a intuição.

No presente trabalho, calculou-se os valores iniciais segundo BARD (1974) e com base no item anterior (4.1.i). Para cada parâmetro foi fixado um intervalo e fez-se todas as combinações possíveis calculando em cada caso a soma dos quadrados dos desvios. Através da menor soma dos çuadrados dos desvios, selecionando-se os valores iniciais dos parâmetros.

\subsection{Ajustamento aos modelos.}

Foram feitos os ajustamentos dos dados aostrês modelos. Na Tabela 4, a seguir é apresentada a análise de va riāncia, em parcelas sub-divididas, dos dados apresentados na Tabela ll, do apêndice.

Vê-se pela análise da Tabela 4 que os efeitos de variedade e da interação ExV foram altamente significatí vos. Isto indica que é conveniente fazer-se o desdobramento da análise conjunta o qual é apresentado a seguir na Tabela 5 
TABELA 4 - Análise de variāncia em parcelas sub-divididas.

\begin{tabular}{lrrrr}
\hline Causas de Var. & G.L. & SQ & QM & $F$ \\
\hline Blocos (B) & 2 & 0,2715 & 0,1357 & \\
Variedade (V) & 7 & 95,8755 & $\begin{array}{r}13,6965 \\
0,3377\end{array}$ & $40,56 * *$ \\
Residuos (a) & 14 & 4,7279 & \\
\hline Parcela & 23 & 100,8751 & & \\
\hline Epoca (E) & 5 & 392,9770 & 78,5954 & $323,55 * *$ \\
Interação (ExV) & 35 & 21,7677 & 0,6219 & $2,56 * *$ \\
Residuo (b) & 80 & 19,4331 & 0,2429 & \\
\hline Total & 143 & 535,0531 & & \\
\hline
\end{tabular}

TABELA 5 - Análise de variância relativa ao desdobramento da interação ExV. Epoca dentro de variedades.

\begin{tabular}{lcrrr}
\hline Causas de Var. & G.L. & SQ & QM & $F$ \\
\hline Blocos & 2 & 0,2715 & 0,1357 & \\
Variedades (V) & 7 & 95,8755 & 13,6965 & $40,56 * *$ \\
Residuo (a) & 14 & 4,7279 & 0,3377 & \\
\hline Parcela & 23 & 100,8751 & & \\
\hline Epoca a. Var. 1 & 5 & 29,7886 & 5,9577 & $22,20 * *$ \\
Epoca d. Var. 2 & 5 & 36,4347 & 7,2869 & $30,00 * *$ \\
Epoca d. Var. 3 & 5 & 48,0763 & 9,6152 & $39,58 * *$ \\
Epoca d. Var. 4 & 5 & 80,4128 & 16,0825 & $66,21 * *$ \\
Epoca d. Var. 5 & 5 & 56,3548 & 11,2709 & $46,40 * *$ \\
Epoca d. Var. 6 & 5 & 59,2087 & 11,8417 & $48,75 * *$ \\
Epoca d. Var. 7 & 5 & 68,4240 & 13,6848 & $56,34 * *$ \\
Epoca d. Var. 8 & 5 & 36,0446 & 7,2089 & $29,68 * *$ \\
Residuo (b) & 80 & 19,4331 & 0,2429 & \\
\hline Total & 143 & 535,0531 & & \\
\hline
\end{tabular}


Pela Tabela 5 vê-se que o desdobramento daépoca dentro de variedade resultou em valor de F altamente signi ficativo, então foi feita a anālise de regressão. Para tanto é necessário fazerem-se as estimativas dos parâmetros dostrês modelos apresentada a seguir.

Modelo Segunda Aproximação de Mitscherlich

Como este modelo é não linear nos parâmetros, suas estimativas foram calculados pelo método de MARQUARDT (1963), descrito em (3.2.1.1.2.). Sendo que os resultados ob tidos estão apresentados na Tabela 6 .

TABELA 6 - Estimativas dos Parâmetros e o Número de Interações do modelo: $\hat{Y}=\hat{A}\left[1-10^{-\hat{C}}(x+\hat{b})\right] 10-\hat{k}(x+\hat{b})^{2}$

\begin{tabular}{lccccc}
\hline Variedades & Iterações & $\AA$ & $\hat{\mathrm{c}}$ & $\hat{\mathrm{b}}$ & $\hat{\mathrm{k}}$ \\
\hline CB40-13 & 18 & 20,5170365 & 0,1566111 & 1,9101964 & 0,0011232 \\
CB41-76 & 22 & 23,5619641 & 0,0771137 & 2,6344793 & 0,0014460 \\
CB53-98 & 35 & 24,1039578 & 0,0738867 & 2,4749882 & 0,0013292 \\
CB47-355 & 24 & 25,8821566 & 0,0843596 & 1,6244501 & 0,0017164 \\
CP51-22 & 24 & 26,3361644 & 0,0801662 & 1,9143962 & 0,0019372 \\
IAC48/65 & 24 & 26,5804874 & 0,0895819 & 1,5471480 & 0,0024533 \\
IAC52/150 & 4 & 17,9106197 & 0,1983600 & 0,9655491 & 0,0006104 \\
Na56-79 & 29 & 24,8156855 & 0,921987 & 2,5617108 & 0,0013762 \\
\hline
\end{tabular}


Modelo Quadrático

Para este modelo a estimativa dos parâmetros fo ram calculados pelo método dos minimos quadrados, conforme $(3.2 .1 .1 .1$.$) e (3.3.). E os resultados obtidos estão apresen$ tados na Tabela 7 .

TABELA 7 - Estimativa dos Parâmetros do Modelo $\hat{Y}=\hat{A}+\hat{B} x+\hat{C x}^{2}$

\begin{tabular}{lrcc}
\hline Variedades & $\hat{\mathrm{A}}$ & $\hat{\mathrm{B}}$ & $\hat{\mathrm{C}}$ \\
\hline CB40-13 & 10,9060 & 2,4608 & $-0,2589$ \\
CB41-76 & 8,7250 & 2,1945 & $-0,2098$ \\
CB53-98 & 8,3300 & 2,2550 & $-0,1979$ \\
CB47-355 & 7,1840 & 3,1714 & $-0,2889$ \\
CP51-22 & 7,9400 & 2,8964 & $-0,2807$ \\
IAC48/65 & 7,5280 & 3,3070 & $-0,3407$ \\
IAC52/150 & 7,8460 & 3,1394 & $-0,2989$ \\
Na56-79 & 10,5470 & 2,3657 & $-0,2309$ \\
\hline
\end{tabular}

Modelo Raiz Quadrada

Como no modelo anterior os parâmetros foramcal culados conforme (3.2.1.1.1.) e (3.3.). E na Tabela 8 estão apresentados os resultados. 
TABELA 8 - Estimativa dos Parâmetros do Modelo $\bar{Y}=\bar{A}+\hat{B} X+\hat{C} \sqrt{X}$.

\begin{tabular}{lcrr}
\hline Variedades & $\hat{\mathrm{A}}$ & $\hat{\mathrm{B}}$ & $\hat{\mathrm{C}}$ \\
\hline CB40-13 & 4,8959 & $-2,3287$ & 10,4393 \\
CB41-76 & 4,9545 & $-1,2982$ & 7,0970 \\
CB53-98 & 3,7142 & $-1,4131$ & 8,0060 \\
CB47-355 & 1,1110 & $-1,9493$ & 10,8642 \\
CP51-22 & 2,2640 & $-1,9994$ & 10,2775 \\
IAC48/65 & 0,2168 & $-2,7843$ & 12,9969 \\
IAC52/150 & 0,5792 & $-2,5060$ & 12,4588 \\
Na56-79 & 5,1484 & $-1,9191$ & 9,3577 \\
\hline
\end{tabular}

Como já foram estimados os valores dós parâmetros dos três modelos, é possível fazer o cálculo da análise da variância levando-se em conta a regressão conforme (3.2.1.3.). Para uma maior simplicidade apresentam-se os valores de $F$ edo coeficiente de determinação $\left(R^{2}\right)$, na Tabela 9.

Comparando-se as análises de regressão da Tabe la 9, notou-se que o modelo quadrático apresentou valores sig

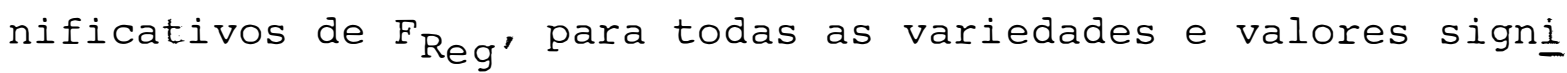
ficativos de F Desv.Reg em menor nümero que os demais métodos. Ademais, os valores dos coeficientes de determinação $\left(\mathrm{R}^{2}\right)$ calculados com base nos resultados obtidos atra vés do modelo quadrático, foram em geral maiores que os coefi cientes de determinação inerentes aos outros dois modelos. 
Estes fatos, sem dúvida, podem sugerir melhor adequação do modelo quadrático aos dados analisados.

iva Tabela 10 apresenta-se para os três modelos a produção máxima bem como o seu respectivo mês.

A produção máxima de sacarose foi aproximadamen te igual para os très modelos. Entretanto, a obtenção desses máximos dá-se, quase sempre antes no modelo quadrático, depois na raiz quadrada, ficando a segunda aproximação de Mitscherlich num ponto intermediário.

Os gráficos das très equações para cada variedade estão no apêndice. Pelos gráficos vê-se que o modelo que mais se aproximou dos dados foi o modelo quadrático confirman do o resultado sugerido na discussão da Tabela 9. Nota-se ain da neste modelo uma queda mais rápida que os outros dois, o que prëjudica qualquer extrapolação [ P IMENTEL GOMES eNOGUEIRA [1975]. Desse modo essa ação não é indicada. Quanto a segun da aproximação de Mitscherlich, apesar de ter uma adaptação um pouco pior que o quadrático, não foge muito do real observado. Este modelo não apresenta muitos problemas quanto à ex trapolação. O modelo raiz quadrada tem uma adaptação pior que os demais, fugindo principalmente nos ültimos pontos. Também apresenta um ponto de máximo mais deslocado para a direita, ou seja, alcançando esse ponto praticamente no final da safra. 


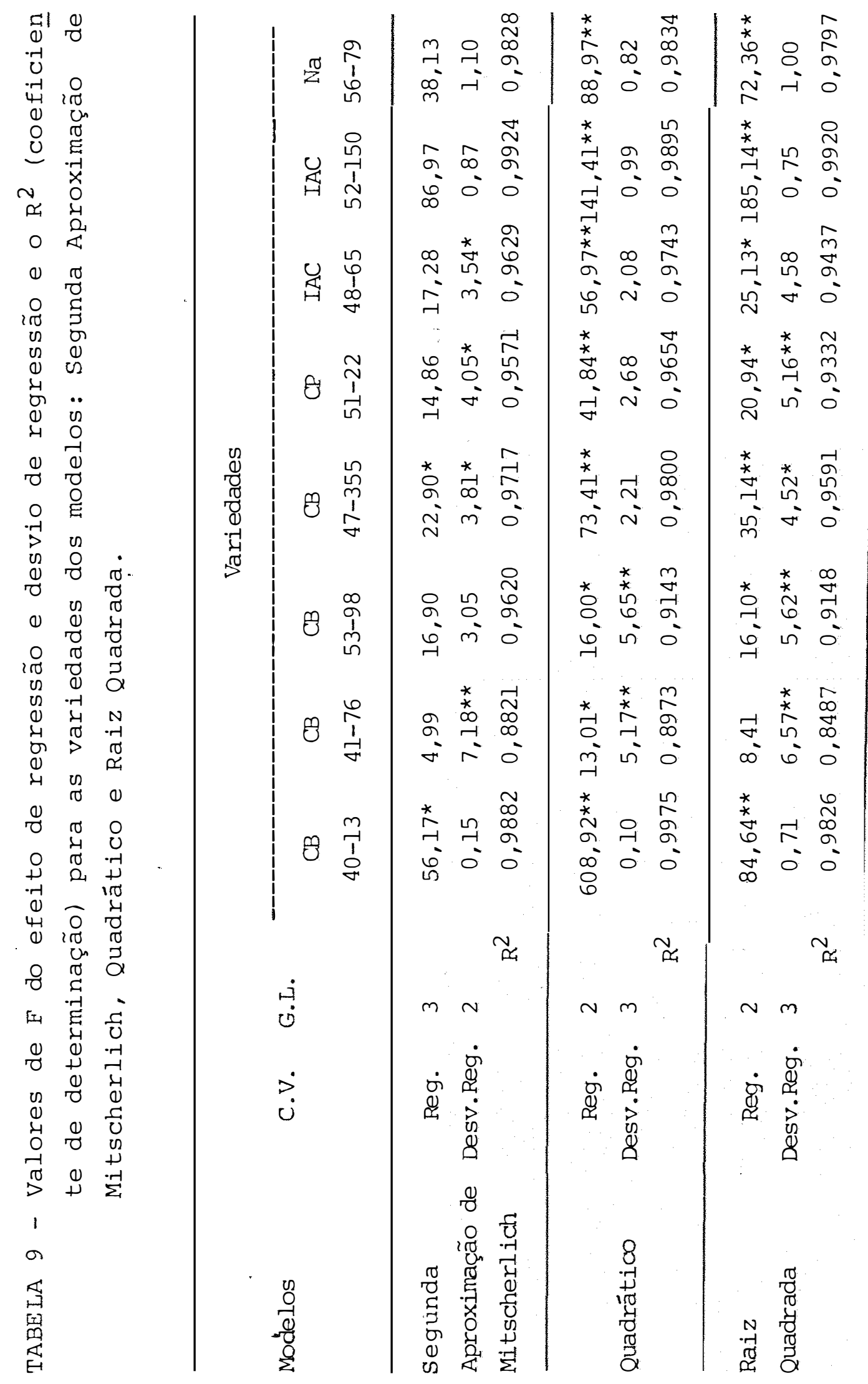




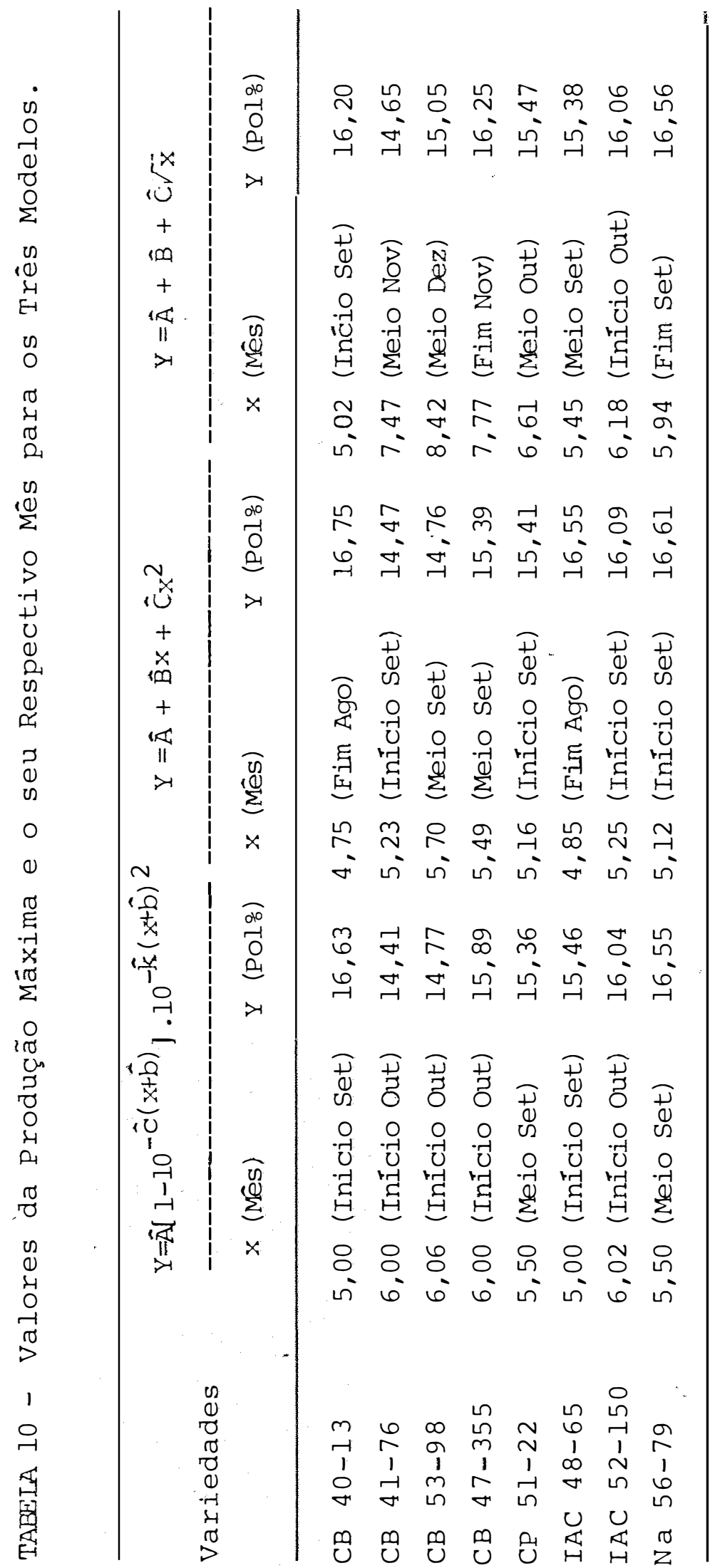




\section{CONCLUSÃO}

Os resultados obtidos na busca do modelo mais adequado para descrever o fenômeno da maturação, nas oito variedades de cana-de-açúcar estudadas, sugerem que:

1) O modelo quadrático foi o que melhor se adaptou ao fenômeno como pode ser visto pelos valores do coeficiente de determinação $\left(R^{2}\right)$, bem como visualmente pelo próprio gráfico.

2) O modelo raiz quadrada foi o que pior se comportou não sendo portanto aconselhável a sua utilização.

3) O modelo segunda aproximação de Mitscherlich, teve uma adaptação inferior ao quadrático.

4) O modelo quadrático apresenta problemas na extrapolação; já na segunda aproximação de Mitscherlich esses problemas são menores. 


\section{BIBLIOGRAFIA}

BARD, Y., 1974. Nonlinear Parameter Estimation. Nova York. Academic Press, Inc., 341 pp.

BOLETIM C.T.C. COPERSUCAR, 1980. 37pp.

BRIEGER, F.O., 1964. Maturação da Cana-de-Açúcar. Boletim Informativo Copereste, Ribeirão Preto, 4(s.n.).

BRIEGER, F.O., 1965. Escolha da Melhor Época para Industrializar a Cana. Boletim Informativo Copereste, Ribeirão Pre to, 4 (s.n.).

BIEGER, F.O., 1967. Açúcar por Hectare. Boletim Informativo Copereste, Ribeirão Preto, 5 (s.n.).

HARTLEY, H.O., 1961. The Modified Gauss-Newton Method for the Fitting of Non-Linear Regression Function by Least Squares. Technometrics, Richmond, 3: 269-280.

MARQUARDT, D.W., 1963. An Algorithm for Least Squares. Estimation of Non-Linear Parameters. Journal Society for Indus trial and Applied Mathematics, Filadelfia, 1l: 431-441. 
MIOQUE, J., 1968. Aumento de Produtividade das Usinas de Açúcar. Boletim Informativo Copereste, Ribeirão Preto, $\underline{4}(\mathrm{~s} \cdot \mathrm{n}$.

OLIVEIRA, E.R. de, C. CRUCCIANI, A.I. BASSINELLO e D. BARBIN, 1974a. Algumas Características Tecnológicas de 15 Varieda des de Cana-de-Açúcar (Resultados Preliminares para CanaPlanta). Brasil Açucareiro, Rio de Janeiro 84(I): 34-43.

OLIVEIRA, E.R. de, C. CRUCCIANI, A.I., BASSINELLO e D. Barbin, 1974b. Algumas Características Tecnológicas de 15 Varieda des de Cana-de-Açúcar (Resultados Preliminares para CanaSoca). Brasil Açucareiro, Rio de Janeiro 84 (3): 22-32.

PIMENTEL GOMES, F. e E. MALAVOLTA, 1949. Considerações Matemāticas sobre a Lei de Mitscherlich. Boletim nọ $\underline{3}$, E.S.A. "Luiz de Queiroz", Piracicaba, $24 \mathrm{pp}$.

PIMENTEL GOMES, F. e I.R. NOGUEIRA, 1975. Extrapolação ou Projeção: Uma Técnica Difícil e Perigosa. Ciência e cultú ra 29: $1386-1389$.

SALVIANO, A., 1973. Aspectos Metodológicos de la Construction de Modelos Estatísticos em las Ciências Sociales Chapingo, $264 \mathrm{pp}$. (Dissertação de Mestrado).

SILVA, M.A.P. da, 1978. Segunda Aproximação de Mitscherlich $Y=A\left[1-10^{-C(x+b)}\right] 10^{-k(x+b) 2}$, Aplicada à Adubação Mineral. Piracicaba, ESALQ/USP, 96pp. (Dissertação de Mestrado).

SILVA, M.A.P. da, 1980. Métodos de Estimação dos Parâmetros do Modelo $Y=A\left[1-10^{-c x} \cdot 10^{-k x^{2}}\right]+e$. Aplicação a dados de Maturação da Cana-de-Açúcar. Piracicaba, ESALQ/USP, 85pp. (Tese de Doutorado). 
49.

APENDICE 
TABELA 11 - Resultados analiticos de Pol c cana, obtidos pela Usina São João, em Araras (SP), em 1979.

Variedade Amos Maio Junho Julho Agosto Setembro Outubro

\begin{tabular}{cccccccc}
\hline & 1 & 12,59 & 14,62 & 15,74 & 16,15 & 17,04 & 16,23 \\
CB 40-13 & 2 & 13,46 & 15,00 & 16,26 & 16,80 & 16,39 & 16,16 \\
& 3 & 13,17 & 15,09 & 15,75 & 16,41 & 17,34 & 16,49 \\
& M & 13,07 & 14,90 & 15,92 & 16,45 & 16,92 & 16,29
\end{tabular}

\begin{tabular}{|c|c|c|c|c|c|c|c|}
\hline & 1 & 15,03 & 11,54 & 13,33 & 14,83 & 14,78 & 14,35 \\
\hline \multirow[t]{4}{*}{ CB $41-76$} & 2 & 10,54 & 10,97 & 12,96 & 14,45 & 13,98 & 14,14 \\
\hline & 3 & 9,76 & 12,26 & 13,32 & 15,44 & 14,21 & 14,13 \\
\hline & $\mathrm{M}$ & 11,11 & 11,59 & 13,20 & 14,91 & 14,32 & 14,21 \\
\hline & 1 & 10,35 & 12,31 & 13,10 & 14,32 & 15,62 & 15,18 \\
\hline \multirow[t]{4}{*}{ CB53-98 } & 2 & 9,90 & 12,79 & 13,09 & 14,36 & 14,70 & 14,58 \\
\hline & 3 & 10,58 & 12,35 & 12,24 & 13,86 & 14,64 & 14,01 \\
\hline & M & 10,28 & 12,48 & 12,81 & 14,18 & 14,99 & 14,59 \\
\hline & 1 & 10,15 & 12,23 & 13,37 & 15,45 & 16,87 & 15,28 \\
\hline \multirow[t]{3}{*}{ CB $47-355$} & 2 & 9,95 & 12,52 & 13,52 & 15,69 & 15,86 & 15,52 \\
\hline & 3 & 10,51 & 12,36 & 13,82 & 16,37 & 15,96 & 15,82 \\
\hline & M & 10,20 & 12,37 & 13,57 & 15,50 & 16,23 & 15,54 \\
\hline
\end{tabular}

cont. 
Tabela 11 - Cont.

\begin{tabular}{|c|c|c|c|c|c|c|c|}
\hline Variedade & $\frac{\text { Amos }}{\text { trá }}$ & Maio & Junho & Julho & Agosto & Setembro & outubro \\
\hline & 1 & 10,66 & 11,92 & 13,58 & 15,37 & 13,92 & 15,43 \\
\hline \multirow[t]{4}{*}{ CP51-22 } & 2 & 10,77 & 12,04 & 13,86 & $16 ; 21$ & 16,77 & 15,14 \\
\hline & 3 & 11,09 & 12,52 & 14,17 & 15,19 & 15,41 & 14,71 \\
\hline & M & 10,84 & 12,16 & 13,87 & 15,59 & 15,37 & 15,09 \\
\hline & 1 & 10,66 & 12,56 & 13,65 & 15,69 & 15,54 & 14,55 \\
\hline \multirow[t]{4}{*}{ IAC48/65 } & 2 & 10,49 & 12,96 & 14,30 & 16,05 & 15,30 & 14,94 \\
\hline & 3 & 10,79 & 12,47 & 13,83 & 15,71 & 15,87 & 15,45 \\
\hline & M & 10,65 & 12,66 & 13,93 & 15,82 & 15,57 & 14,98 \\
\hline & 1 & 9,62 & 13,10 & 15,57 & 15,60 & 15,85 & 15,75 \\
\hline \multirow[t]{4}{*}{ IAC52/150 } & 2 & 11,05 & 12,83 & 14,42 & 15,18 & 16,17 & 15,83 \\
\hline & 3 & 11,07 & 13,55 & 14,80 & 14,97 & 16,08 & 16,48 \\
\hline & M & 10,58 & 12,99 & 14,93 & 15,25 & $16,0.3$ & 16,02 \\
\hline & 1 & 11,94 & 14,37 & 15,84 & 16,06 & 16,48 & 16,54 \\
\hline \multirow[t]{3}{*}{ Na56-79 } & 2 & 12,96 & 14,60 & 14,79 & 16,05 & 17,23 & 15,86 \\
\hline & 3 & 12,94 & 14,78 & 15,62 & 16,26 & 16,96 & 16,60 \\
\hline & M & 12,61 & 14,58 & 16,42 & 16,12 & 16,89 & 16,33 \\
\hline
\end{tabular}


TABELA 12 - Valores Observados e suas Estimativas através dos três Modelos.

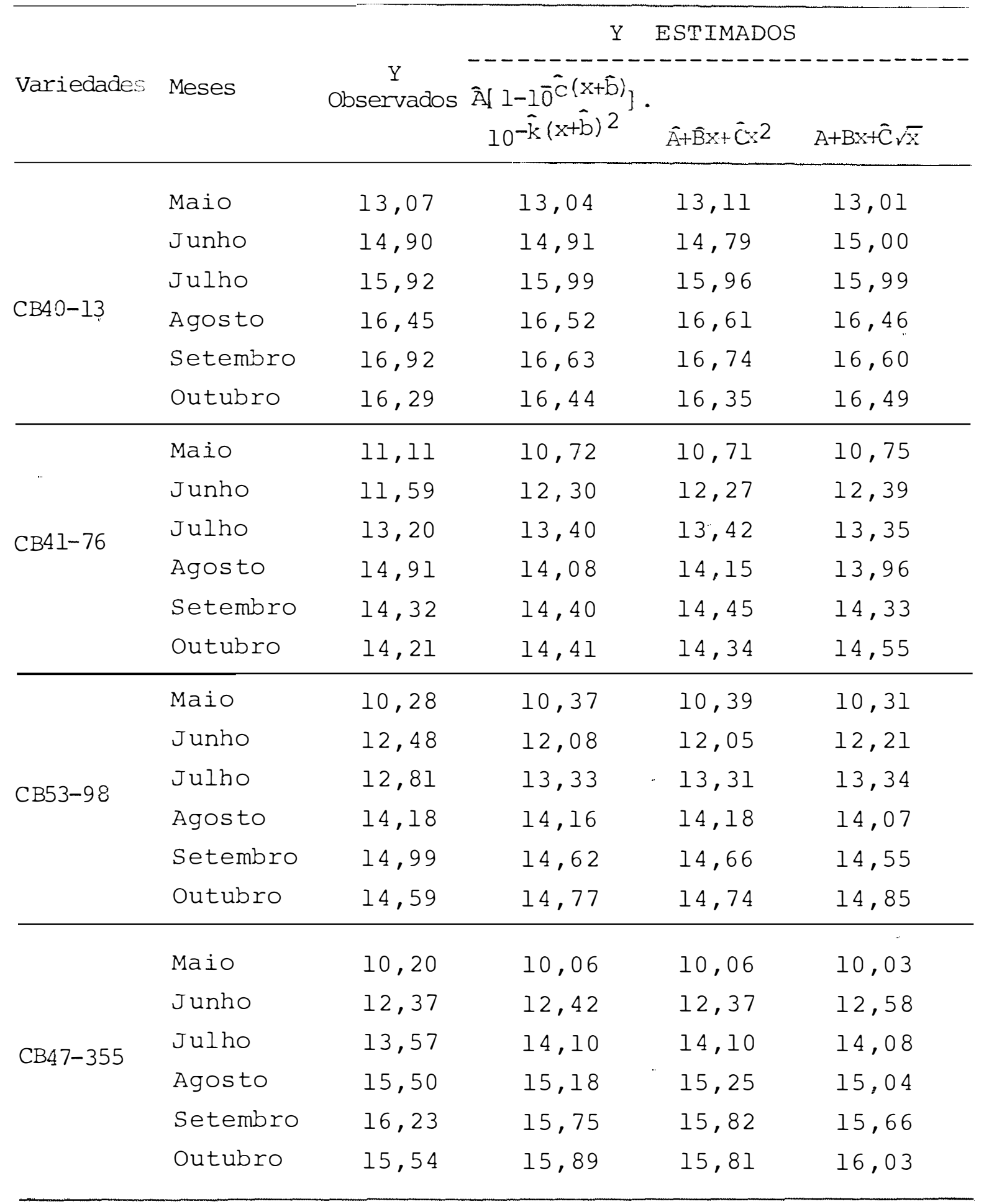


TABELA 12 - Cont.

Y ESTIMADOS,

Variedades Meses

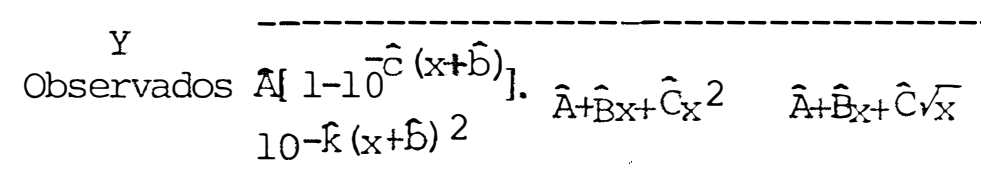

\begin{tabular}{|c|c|c|c|c|c|}
\hline \multirow{6}{*}{ CP51-22 } & Maio & 10,84 & 10,55 & 10,56 & 10,54 \\
\hline & Junho & 12,16 & 12,65 & 12,61 & 12,80 \\
\hline & Julho & 13,87 & 14,10 & 14,10 & 14,07 \\
\hline & Agosto & 15,59 & 14,97 & 15,03 & 14,82 \\
\hline & Setembro & 15,37 & 15,34 & 15,40 & 15,25 \\
\hline & Outubro & 15,09 & 15,30 & 15,21 & 15,44 \\
\hline \multirow{6}{*}{ IAC $48 / 65$} & Maio & 10,54 & 10,47 & 10,49 & 10,43 \\
\hline & Junho & 12,66 & 12,85 & 12,78 & 13,03 \\
\hline & Julho & 13,93 & 14,39 & 14,38 & 14,38 \\
\hline & Agosto & 15,82 & 15,22 & 15,30 & 15,07 \\
\hline & Setembro & 15,57 & 15,46 & 15,55 & 15,36 \\
\hline & Outubro & 14,98 & 15,21 & 15,10 & 15,35 \\
\hline \multirow{6}{*}{ IAC52/150 } & Maio & 10,58 & 10,55 & 10,69 & 10,53 \\
\hline & Junho & 12,99 & 13,13 & 12,93 & 13,19 . \\
\hline & Julho & 14,93 & 14,66 & 14,57 & 14,64 \\
\hline & Agosto & 15,25 & 15,51 & 15,62 & 15,47 \\
\hline & Setembro & 16,03 & 15,92 & 16,07 & 15,91 \\
\hline & Outubro & 16,02 & 16,04 & 15,92 & 16,06 \\
\hline \multirow{6}{*}{$\mathrm{Na} 56 / 79$} & Maio & 12,61 & 12,65 & 12,68 & 12,59 \\
\hline & Junho & 14,58 & 14,41 & 14,35 & $14,54$. \\
\hline & Julho & 15,42 & 15,59 & 15,57 & 15,60 \\
\hline & Agosto & 16,12 & 16,27 & 16,32 & 16,19 \\
\hline & Setembro & 16,89 & 16,55 & 16,60 & 16,48 \\
\hline & Outubro & 16,33 & 16,45 & 16,43 & 16,56 \\
\hline
\end{tabular}




\section{PROGRAMA 1}

C

C

C

C

C

C

C

MÉTODO DE MARQUARDT

CÁLCULO DAS ESTIMATIVAS DOS PARÂMETROS DA FUNÇÃO $\mathrm{Y}=\mathrm{A} *(1-10 * *(-\mathrm{C}(\mathrm{X}+\mathrm{B})) * 10 * *(-\mathrm{K}(\mathrm{X}+\mathrm{B}) * * 2)$

$A O, C O, B O, P K O$ SÃO OS VALORES INICIAIS DE $A, C, K, B$

N NUMERO DE OBSERVAÇÕES

NI NUMERO DE EQUAÇÕES

UTILIZA A SUBROTINA PIRI 1

DIMENSION A (49), P (49), B (49), L ( 7) ,M(7), O(10), XI (20), $\mathrm{X}(50), \mathrm{Y}(50), \mathrm{YES}$

I (T) 50, DESV(50), $\operatorname{VALI}(20), \operatorname{SN}(50), \operatorname{YYEST}(20), \operatorname{DIF}(20)$, XEST (20), DIFE (20)

$1001 \quad \operatorname{PEAD}(2,100) \mathrm{N}, \mathrm{Nl}, \mathrm{AO}, \mathrm{CO}, \mathrm{BO}, \mathrm{PKO}$

100 FORMAT $(215,4 \mathrm{~F} 10.0)$

$\operatorname{READ}(2,101)(X(I), I=1, N)$

101 FORMAT $(10 \mathrm{~F} 8,0)$

$\operatorname{READ}(2,102)(\mathrm{Y}(\mathrm{I}), \mathrm{I}=1, \mathrm{~N})$

102 FORMAT (10F8.0)

$\mathrm{N} 2=\mathrm{N} I * \mathrm{~N} 1$

ITER $=0$

IP $=1$

SLAMB $=0.01$

$500 \quad \mathrm{SQ}=0$

DO I I $=1, \mathrm{~N}$

$\mathrm{YY} I=\mathrm{X}(\mathrm{I})+\mathrm{B} 0$

$Y Y 2=-C O * Y Y I$

YY $3=-P K O *(Y Y I$ * * 2$)$

$Y Y 4=10 . * * Y Y 2$

YY $5=10 . * * Y Y 3$

$\mathrm{YY} 6=\mathrm{AO} *(1-\mathrm{YY} 4)$

$\operatorname{YYEST}(I)=Y Y 6$ *YY 5

$\operatorname{DIF}(I)=Y(I)-Y Y E S T(I)$

$1 \mathrm{SQ}=\mathrm{SQ}+\mathrm{DIF}(\mathrm{I}) *$ * 2

WRITE $(3,200)$ SQ,ITER 
200 FORMAT $\left(5 X, ' S Q=', F 25,8,5 X\right.$ I ITEPF $\left._{2}=', I 4\right)$

WRITE $(3,201) \mathrm{AO}, \mathrm{CO}, \mathrm{BO}, \mathrm{PKO}$

201 FORMAT $\left(5 \mathrm{X},{ }^{\prime} \mathrm{AO}={ }^{\prime}, \mathrm{F} 25.8,{ }^{\prime} \mathrm{CO}={ }^{\prime}, \mathrm{F} 15.8,{ }^{\prime} \mathrm{BO}={ }^{\prime}, \mathrm{F} 15.8\right.$, $' \mathrm{PKO}=$ ', $\mathrm{F} 15.8$ )

DO $93 \mathrm{~J}=1, \mathrm{Nl}$

$93 \Omega(J)=0$

DO $94 \mathrm{~J}=1, \mathrm{~N} 2$

$94 \mathrm{~A}(\mathrm{~J})=0$

C

CALCULO DA MATRIZ N

DO $95 \mathrm{I}=1, \mathrm{~N}$

$\mathrm{CIDA}=10 \cdot * \star(-\mathrm{CO} *(\mathrm{X}(\mathrm{I})+\mathrm{BO}))$

$\mathrm{CASO}=10 \cdot * \star(-\mathrm{PKO} *(\mathrm{X}(I)+\mathrm{BO} * \star 2)$

$\mathrm{F} I=(1-\mathrm{CIDA}) * \mathrm{CASO}$

$\mathrm{F} 2=(\mathrm{X}(\mathrm{I} ; \mathrm{BO}) * \mathrm{AO} * \mathrm{CIDA} * \mathrm{CASO} * \mathrm{AIOG}(10)$.

$\mathrm{F} 3=\mathrm{AO} * \mathrm{CASO} * \mathrm{ALOG}(10 \cdot) *(-2 * \mathrm{PKO} *(\mathrm{X}(\mathrm{I})+\mathrm{BO})+(\mathrm{CO}+2 * \mathrm{PKO}$ * $(X(I)+B O)) * C I D A)$

$\mathrm{F} 4=-(\mathrm{X}(\mathrm{I})+\mathrm{BO}) * * 2 * \mathrm{AO} *(1-\mathrm{CIDA}) * \mathrm{CASO}$ \# F.LG $(10$.

$\mathrm{A}(I)=\mathrm{A}(I)+\mathrm{F} I * \star 2$

$A(2)=A(2)+F I * F 2$

$\mathrm{A}(3)=\mathrm{A}(3)+\mathrm{Fl}$ * F 3

$A(4)=A(4)+F I * F 4$

$A(6)=A(6)+F 2 * \star 2$

$A(7)=A(7)+F 2 * F 3$

$A(8)=A(8)+F 2{ }^{\star} F 4$

$A(I I)=A(I I)+F 3 * \star 2$

$\mathrm{A}(12)=\mathrm{A}(12)+\mathrm{F} 3^{*} \mathrm{~F} 4$

$\mathrm{A}(16)=\mathrm{A}(16)+\mathrm{F} 4 * \star 2$

C

CÁLCULO DO VETOR $Q$

$Q(I)=Q(I)+(Y(I)-A O * F I) * F I$

$Q(2)=Q(2)+(Y(I)-A O * F I) * F 2$

$Q(3)=Q(3)+(Y(I)-A O * F I) * F 3$

$95 \mathrm{Q}(4)=\mathrm{Q}(4)+(\mathrm{Y}(\mathrm{I})-\mathrm{AO}$ *FI)*F4

C

MATRIZ A

$A(I)=2 * A(I)$

$A(2)=2 * A(2)$ 


$$
\begin{aligned}
& A(3)=2 * A(3) \\
& A(4)=2 * A(4) \\
& A(5)=A(2) \\
& A(6)=2 * A(6) \\
& A(7)=2 * A(7) \\
& A(8)=2 * A(8) \\
& A(9)=A(3) \\
& A(10)=A(7) \\
& A(11)=2 * A\left(\begin{array}{ll}
l & 1
\end{array}\right) \\
& A(12)=2 * A(12) \\
& A(13)=A(4) \\
& A(14)=\dot{A}(8) \\
& A(15)=A(12) \\
& A(16)=2 * A(16) \\
& \text { C VETOR } \\
& Q(I)=-2^{*} Q(I) \\
& Q(2)=-2 * Q(2) \\
& Q(3)=-2 * Q(3) \\
& \mathrm{Q}(4)=-2 * \mathrm{Q}(4) \\
& \text { C } \\
& \text { CALCULO DA MATRIZ } \mathrm{B}=\mathrm{N}+\mathrm{LP} \\
& \text { IF. }(A(I)) 2,3,2 \\
& 2 \quad P(I)=\operatorname{ABS}(\mathrm{A}(I)) \\
& \text { GO TO } 300 \\
& 3 \quad P(1)=1 \\
& 300 \quad \operatorname{IF}(A(6)) 4,5,4 \\
& 4 \quad P(6)=\operatorname{ABS}(A(6)) \\
& \text { GO TO } 301 \\
& 5 \quad P(6)=1 \\
& 301 \operatorname{IF}(A(11)) 6,7,6 \\
& 6 \quad P(11)=\operatorname{ABS}(A(11)) \\
& \text { GO TO } 302 \\
& 7 \quad P(11)=1 \\
& 302 \operatorname{IF}(A(I 6)) 8,9,8 \\
& 8 \quad \mathrm{P}(16)=\mathrm{ABS}(\mathrm{A}(16)) \\
& \text { GO TO } 303 \\
& 9 \quad P(16)=1
\end{aligned}
$$




$$
\begin{aligned}
& 303 \quad P(2)=0 \\
& P(3)=0 \\
& P(4)=0 \\
& P(5)=0 \\
& P(7)=0 \\
& P(8)=0 \\
& P(9)=0 \\
& P(10)=0 \\
& P(12)=0 \\
& P(13)=0 \\
& P(14)=0 \\
& P(15)=0 \\
& \text { DO } 28 I=I, N 2 \\
& 28 \mathrm{~B}(\mathrm{I})=\mathrm{A}(\mathrm{I})+\mathrm{SLAMP} * \mathrm{P}(\mathrm{I}) \\
& \text { DO } 5000 \quad I=1,16 \\
& 5000 \quad \mathrm{SN}(I)=\mathrm{B}(I) \\
& \text { C } \\
& \text { INVERSÃO DA MATRIZ B } \\
& \text { CALL MINV }(B, N I, D, L, M) \\
& \text { DO TO }(501,502) \text {, IP } \\
& 501 \quad \text { I } I=0 \\
& \text { DO } 29 \mathrm{I}=1, \mathrm{~N} I \\
& \text { C CALCULO DO VETOR SOLUÇÃO D } \\
& X I(I)=0 \\
& \mathrm{I} I=\mathrm{I} I+\mathrm{N} I \\
& \text { DO } 29 \mathrm{~J}=1, \mathrm{NI} \\
& \text { I } 2=I 1-N I \\
& \mathrm{I} 3=\mathrm{J}+\mathrm{I} 2 \\
& 29 X I(I)=X I(I)+B(I 3) *(-Q(J)) \\
& \mathrm{VI}=\mathrm{XI}(1){ }^{*} \mathrm{Q}(1)+\mathrm{XI}(2) * \mathrm{Q}(2)+\mathrm{XI}(3) * \mathrm{Q}(3)+\mathrm{XI}(4) * \mathrm{Q}(4) \\
& \text { C } \\
& \text { CALLULO DE NOVAS ESTIMATIVAS DOS PARÂMETROS } \\
& \mathrm{AAI}=\mathrm{AO}+\mathrm{XI}(1) \\
& \mathrm{CCl}=\mathrm{CO}+\mathrm{XI}(2) \\
& \mathrm{BBI}=\mathrm{BO}+\mathrm{XI}(3) \\
& \operatorname{IF}(\mathrm{BBI}) 78,79,79 \\
& 78 \mathrm{BBI}=\mathrm{BO}-0.1 * \mathrm{BO}
\end{aligned}
$$


79

CONTINUE

$\mathrm{PPKI}=\mathrm{PKO}+\mathrm{XI}(4)$

IF (PPKI) $88,89,89$

$88 \quad \mathrm{PPKI}=\mathrm{PKO}-0 . I * \mathrm{PKO}$

89 CONTINUE

$\mathrm{SQO}=0$

C

VERIFICA-SE A SOLUÇ $\widetilde{A} O$ D E UM PASSO ACEITÁVEL

DO $10 \quad I=I, N$

$\mathrm{XXI}=\mathrm{X}(\mathrm{I})+\mathrm{BB} I$

$\mathrm{XX} 2=-\mathrm{CC} I * \times \times 1$

$\mathrm{XX} 3=-\mathrm{PPKI}$ * $(\mathrm{XXI} * * 2)$

$\mathrm{XX} 4=10 . \star \star \times \times 2$

$\mathrm{XX5} 5=10 . * * \times \times 3$

$\mathrm{XX6}=\mathrm{AA} 1 *(1 .-\mathrm{XX} 4)$

$\operatorname{XEST}(I)=X \times 6 * \times X 5$

$\operatorname{DIFE}(I)=Y(I)-X E S T(I)$

$10 \mathrm{SQO}=\mathrm{SQO}+\mathrm{DIFE}(\mathrm{I}) *$ * 2

IF (SQO-SQ) $11,12,12$

II CONTINUE

$\operatorname{IF}(0.1 *$ SLAMB -0.0000001$) 80,80,81$

$80 \quad \mathrm{SLAMB}=0.0000001$

GO TO 82

$81 \mathrm{SLAMB}=0,1 *$ SLAMB

GO TO 82

12 CONTINUE

CÁLCULO DE RO PARA OBTER UM PASSO ACEITÁVEL RMIN $=0.0000000001$

$\mathrm{RMAX}=10 . * * 20$

$\operatorname{VALI}(I)=A O$

$\operatorname{VALI}(2)=\mathrm{CO}$

$\operatorname{VALI}(3)=\mathrm{B}$ ?

$\operatorname{VALI}(4)=\mathrm{PKO}$

DO $19 \mathrm{~J}=5,16$

$X I(J)=0$ 
$\operatorname{VALI}(J)=0$

$J=1$

CALL PIRII (SQ, XI, X, Y, N , VI , VALI, RMAX, RMIN , J , ROI)

$\mathrm{AAI}=\mathrm{AO}+\mathrm{ROI} * \mathrm{XI}(1)$

$\mathrm{CCl}=\mathrm{CO}+\mathrm{ROI} * \mathrm{XI}(2)$

$\mathrm{BBI}=\mathrm{BO}+\mathrm{ROI} * \mathrm{XI}(3)$

$\operatorname{IF}(\mathrm{BBI}) 61,63,63$

$61 \quad \mathrm{BBI}=\mathrm{BO}-0.1 * \mathrm{BO}$

63 CONTINUE

$\mathrm{PPKI}=\mathrm{PKO}+\mathrm{ROI}$ *XI $(4)$

$\operatorname{IF}(\mathrm{PPKI}) 71,73,73$

$71 \quad \mathrm{PPKI}=\mathrm{PKO}-0.1 * \mathrm{PKO}$

73 CONTINUE

$\mathrm{SLAMB}=10 . * \mathrm{SLAMB}$

82 CONTINUE

C

TESTE DE FINALIZAÇÃO DO PROCESSO

$A X=A B S(A O)$

$E R R O I=(A X+0.001) * 0.0 I$

$\mathrm{CX}=\mathrm{ABS}(\mathrm{CO})$

$E R R O 2=(C X+0.0 .01) * 0.01$

$\mathrm{BX}=\mathrm{ABS}(\mathrm{BO})$

$E R R O 3=(B X+0.001) * 0.01$

$\mathrm{PPX}=\mathrm{ABS}(\mathrm{PKO})$

$\mathrm{ERRO} 4=(\mathrm{PPX}+0.001) * 0.01$

TESTI $=$ ABS $(A A I-A O)$

TEST2 $=$ ABS $(\mathrm{CC} I-C O)$

TEST $3=A B S(B B I-B O)$

TEST $4=$ ABS $($ PPKI-PKO $)$

IF (TESTl-ERROl) 5l, 51, 52

51 IF (TEST2-ERRO2) 53,53,52

53 IF (TEST3-ERRO3) 54,54,52

54 IF (TEST4-ERRO4) 55,55,52

INICIO DE UMA NOVA ITERAÇÃO

$52 \quad \mathrm{AO}=\mathrm{AAI}$.

$\mathrm{CO}=\mathrm{CCl}$ 


$$
\begin{aligned}
& \mathrm{BO}=\mathrm{BBI} \\
& \mathrm{PKO}=\mathrm{PP} \mathrm{Kl} \\
& \text { ITE } \mathrm{R}=\text { ITE } \mathrm{R}+1 \\
& \text { GO TO } 500 \\
& 55 \text { IP }=2 \\
& \text { C } \\
& \text { SOLUÇ } \widetilde{A} O \text { FINAL } \\
& \mathrm{AO}=\mathrm{AAI} \\
& \mathrm{CO}=\mathrm{CCl} \\
& \mathrm{BO}=\mathrm{BBI} \\
& \mathrm{PKO}=\mathrm{PPKI} \\
& \text { GO TO } 500 \\
& 502 \quad S Q D=0 \\
& \mathrm{SQTOT}=0 \\
& \mathrm{COR}=0 \\
& \text { DO } 59 \mathrm{I}=1, \mathrm{~N} \\
& 59 \mathrm{COR}=\mathrm{COR}+\mathrm{Y}(\mathrm{I}) \\
& \mathrm{CORR}=\mathrm{COR} \star 2 / \mathrm{N} \\
& \operatorname{WRITE}(3,2121) \mathrm{CORR} \\
& 2121 \text { FORMAT (IX, ' CORR=',FI5.6) } \\
& \text { DO } 60 \quad I=1, N \\
& \mathrm{YI}=\mathrm{X}(\mathrm{I})+\mathrm{BBI} \\
& Y 2=-C C I * Y I \\
& Y 3=-P P K I \text { * }(Y I \text { * * } 2) \\
& Y 4=10 . * * Y 2 \\
& Y 5=10 . \star \star Y 3 \\
& \mathrm{Y} 6=\mathrm{AAI} \text { * }(1-\mathrm{Y} 4) \\
& \operatorname{YEST}(I)=Y 6 * Y 5 \\
& \operatorname{DESV}(I)=Y(I)-\operatorname{YEST}(I) \\
& \mathrm{SQD}=\mathrm{SQD}+\mathrm{DESV}(I) * \star 2 \\
& 60 \mathrm{SQTOT}=\mathrm{SQTOT}+\mathrm{Y}(\mathrm{I}) * \text { *2 } \\
& S Q P A R=S A T O T-S Q D \\
& \text { R2=SOPAR/SQTOT } \\
& \operatorname{WRITE}(3,6969)
\end{aligned}
$$

6969 FORMATI (20X, 'METODO DE MARQUARDT',//) $\operatorname{WRITE}(3,7878)$ 
7878 FORMAT (1X,15X, 'MATRIZ INVERSA', //)

$\operatorname{WRITE}(3,801)(B(I), I=1,16)$

801 FORMAT $(5 \mathrm{X}, 4 \mathrm{E} 17.7)$

$\operatorname{WRITE}(3,804)$

WRITE $(3,6765)$

6765 FORMAT (IX, 20X, 'MATRIZ HESSIANA', //)

$\operatorname{WRITE}(3,5001)(\mathrm{A}(\mathrm{I}), I=1,16)$

5001 FORMAT $(5 X, 4 E 17.7)$

$804 \quad$ FORMAT $(I X, /)$

$\operatorname{WRITE}(3,804)$

$\operatorname{WRITE}(3,802)(\mathrm{Q}(\mathrm{I}), I=1,4)$

802 FORMAT $(5 \mathrm{X}, \mathrm{E} 17.7, /)$

WRITE $(3,1040)$ SQTOT, SQPAR, SQD

1040 FORMAT (5X,' SQTOT=' ,F25.4, 2X, 'SQPAR=' ,F25.4, 2X, ' $\mathrm{SQDE}=1, \mathrm{~F} 15.4, /$ )

$\operatorname{WRITE}(3,804)$

1050 FOPMAT (I0X,' R2=', EI7.7,//)

$\operatorname{WRITE}(3,199)$

$\operatorname{WRITE}(3,1050) \mathrm{R} 2$

199 FORMAT (/)

WRITE $(3,150) A A 1, C C 1, B B 1, P P K 1, I T E R$

150 FORMAT (5X,' $\mathrm{A}={ }^{\prime}, \mathrm{F} 15,8,2 \mathrm{X},{ }^{\prime} \mathrm{C}={ }^{\prime}, \mathrm{F} 15.8,2 \mathrm{X},{ }^{\prime} \mathrm{B}={ }^{\prime}, \mathrm{F} 15$.

$$
.8,2 \mathrm{X}, ' \mathrm{~K}={ }^{\prime}, \mathrm{F} I 5.8, ' \mathrm{IT}
$$

$4 E R=1, I 3, / / /)$

$\operatorname{WRITE}(3,199)$

$\operatorname{WRITE}(3,750)$

750 FORMAT (5X,' X', IIX,' $\mathrm{Y}^{\prime}, I 5 \mathrm{X}, \mathrm{X}^{\prime} \mathrm{YEST}$ ', I3X,' DESV')

$\operatorname{WRITE}(3,850)$

$850 \quad$ FORMAT $\left(5 X, 52\left({ }^{\prime}-^{\prime}\right)\right)$

DO $72 I=1, N$

$72 \operatorname{WRITE}(3,950) X(I), Y(I), \operatorname{YEST}(I), \operatorname{DESV}(I)$

950 FORMAT (2X,F6.2,3(2X,F15.8))

$\operatorname{WRITE}(3,850)$

CALI $\operatorname{MINV}(A, N I, D, L, M)$

$\operatorname{WRITE}(3,7979)$ 


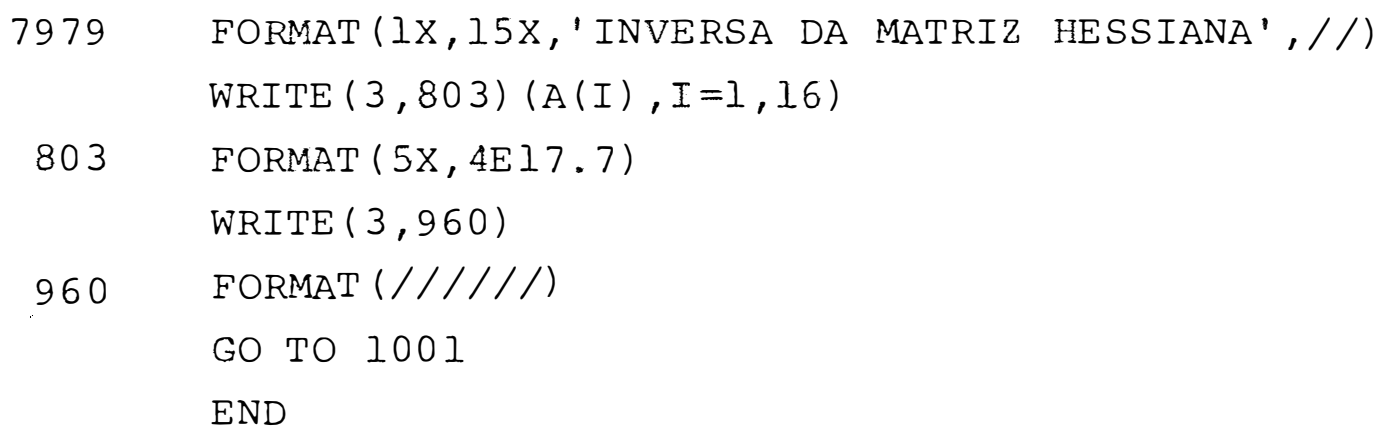

FEATURES SUPPORTED

ONE WORD INTEGERS

EXTENDED PRECISION 
SUBROTINA PIRII

C

C

SUBROTINA PIRII

INTERPOLA O VALOR DE RO PARA A OBTENÇÃO DE UM PASSO ACEITAVEL

SUBROUTINE PIRII (SQ,SOL, X, Y, N , VI, VALI, RMAX, RMIN, $\mathrm{J}, \mathrm{ROI})$

DIMENSION VALI (20), BETAO (20), BETAI (20), SOL (20), $X(50), Y(50), \operatorname{DESV}(20$

1) $\operatorname{ALYES}(20), \operatorname{GAYES}(20), \operatorname{DIFE}(20), \operatorname{EFYES} q 20), \operatorname{DIFE}(20)$

$\mathrm{ROO}=1 \cdot / 2 \cdot * \star \mathrm{J}$

DO $1 \mathrm{I}=1,16$

$\operatorname{BETAO}(I)=\operatorname{VALI}(I)+\mathrm{ROO} * \mathrm{SOL}(\mathrm{I})$

$\operatorname{IF}(\operatorname{BETAO}(3)) 41,42,42$

$41 \quad \operatorname{BETAO}(3)=\operatorname{VALI}(3)-.0.1 * \operatorname{VALI}(3)$

42 CONTINUE

$\operatorname{IF}(\operatorname{BETAO}(4)) 50,51,51$

$50 \quad \operatorname{BETAO}(4)=\operatorname{VALI}(4)-0.1 * \operatorname{VALI}(4)$

51 CONTINUE

$\mathrm{SQO}=0$

DO $2 \mathrm{I}=1, \mathrm{~N}$

$\mathrm{ALFA} I=\mathrm{X}(\mathrm{I})+\mathrm{BETA} 0$

ALFA 2 $=-\operatorname{BETAO}(2) * A L F A I$

$\operatorname{ALFA} 3=-\operatorname{BETAO}(4) *(\operatorname{ALFAl} * \star 2)$

ALFA $4=10 . * \star A L F A 2$

ALFA $5=10 . * * A L F A 3$

ALFA 6 $=\operatorname{BETAO}(1) *(1 .-\operatorname{ALFA} 4)$

$\operatorname{ALYES}(I)=A L F A 6 * A L F A 5$

$\operatorname{DESV}(I)=\mathrm{Y}(I)-\operatorname{ALYES}(I)$

$2 \mathrm{SQO}=\mathrm{SQO}+\operatorname{DESV}(\mathrm{I}) * \star 2$

$\mathrm{ROAST}=\mathrm{V} I$ * $\mathrm{ROO}$ * $* 2 /(2$ * $(\mathrm{VI} * \mathrm{ROO}+\mathrm{SQ}-\mathrm{SQO}))$

IF (SQO-SQ) 3, 4, 4

$4 \quad \mathrm{ROI}=\mathrm{ROO}$

$20 \operatorname{IF}(0.75 *$ ROI-ROAST $) 5,5,6$

$5 \operatorname{IF}(0.25 * \mathrm{RO}-0.75 * \mathrm{RO} 1) 7,7,8$

$7 \quad \mathrm{RO} 2=0.75 * \mathrm{RO}$

GO TO 11 


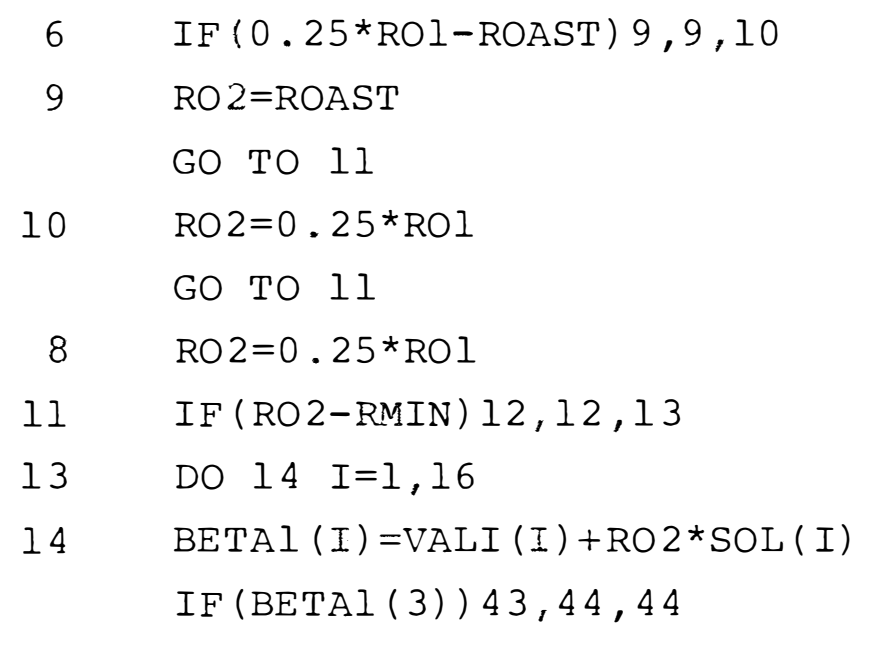

$43 \operatorname{BETAI}(3)=\operatorname{VALI}(3)-0.1 * \operatorname{VALI}(3)$

44 CONTINUE

$\operatorname{IF}(\operatorname{BETAI}(4)) 52,53,53$

$52 \operatorname{BETAI}(4)=\operatorname{VALI}(4)-0.1 * \operatorname{VALI}(4)$

53. CONTINUE

$\mathrm{SQ} 2=0$

DO $15 \mathrm{I}=1, \mathrm{~N}$

GAMAl $=\mathrm{X}(\mathrm{I})+\mathrm{BETAl}(3)$

GAMA2 $=-\operatorname{BETAl}(2) *$ GAMAl

$\operatorname{GAMA} 3=-\operatorname{BETAl}(4) *(\operatorname{GAMAl} * * 2)$

GAMA $4=10 . * \star$ GAMA 2

GAMA $5=10 \cdot * \star$ GAMA 3

GAMA $6=\operatorname{BETAl}(1) *(1 .-\operatorname{GAMA} 4)$

GAYES $(I)=$ GAMA 6 * GAMA 5

$\operatorname{DIF}(I)=Y(I)-\operatorname{GAYES}(I)$

$15 \mathrm{~S} \Omega 2=\mathrm{S} Q 2+\mathrm{DIF}(\mathrm{I}) * \star 2$

$\mathrm{J}=\mathrm{I}+1$

IF (SQ2-SQ) 16,17,17

$17 \quad \mathrm{RO} I=\mathrm{RO} 2$

$\mathrm{ROAST}=\mathrm{V} 1$ * $\mathrm{RO}$ * $* 2 /(2 *(\mathrm{VI} * \mathrm{RO}+\mathrm{SQ}-\mathrm{SQ} 2))$

GO TO 20

$16 \mathrm{ROI}=\mathrm{RO} 2$

12 RETURN

$3 \quad \mathrm{~J}=\mathrm{J} / 2$

IF ( ROAST) $21,21,22$

21 ROAST $=2 *$ ROO 


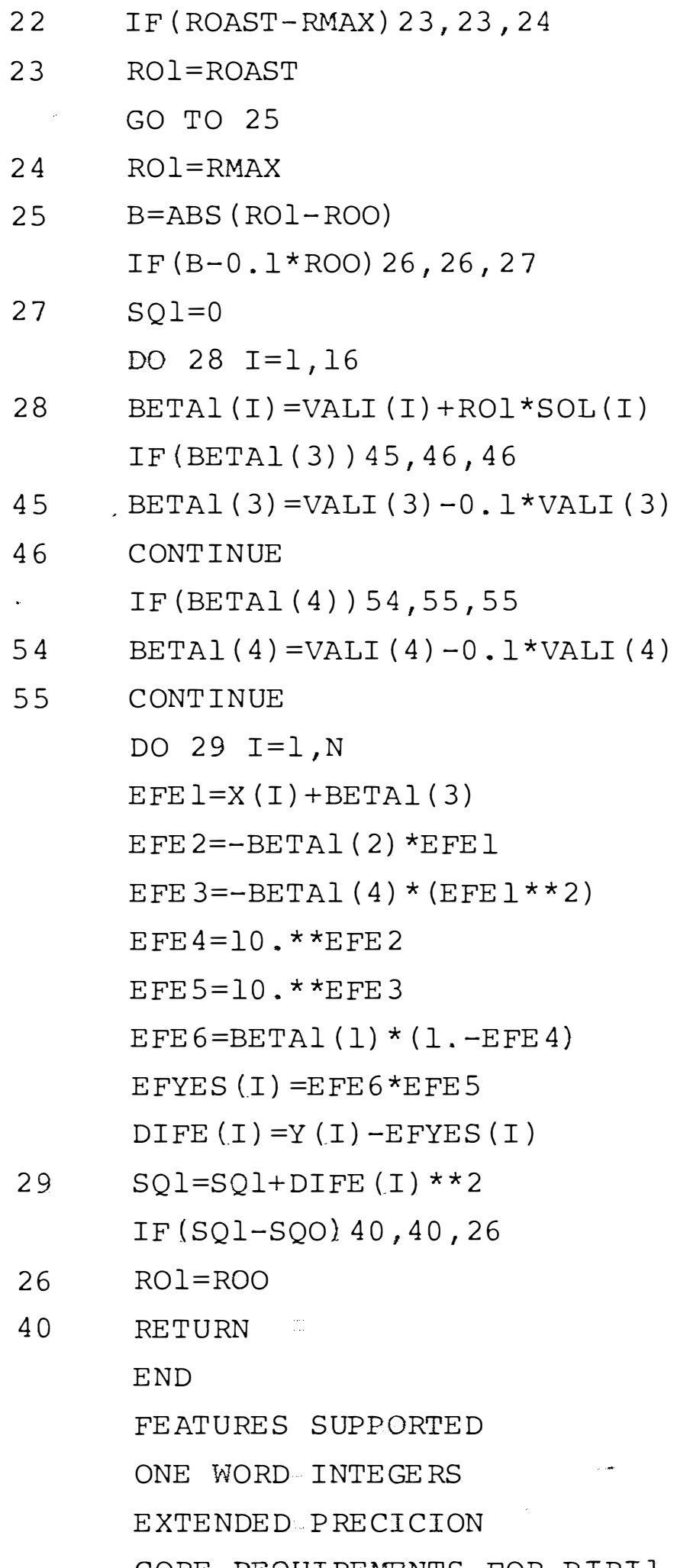

CORE REQUIREMENTS FOR PIRII

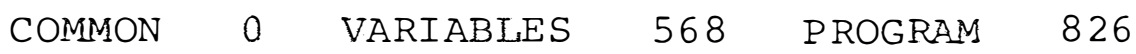


RELATIVE ENTRY POINT AFRESS IS 024E (HEX)

END OF COMPILATION

// DUP

* DEFLETE

PIRII

$\begin{array}{lllllllll}\text { CART ID } 0002 & \text { DB ADDR } & 4668 & \text { DB } & \text { CNT } & 0039\end{array}$

*STORF WS UA PIRII

CART ID 002 OB ADDR 5I7E DB CNT $003 \mathrm{E}$ 


\section{PROGRAMA 2}

C

C

1000

1 FORMAT (IID, 5FIO.0)

$\operatorname{READ}(2,2)(2(I), I=1, N)$

2 FORMAT ( $10 \mathrm{~F} 8,0$

WRITE $(3,10.01) \mathrm{N}, \mathrm{AO}, \mathrm{CO}, \mathrm{BO}, \mathrm{PKO}$

1001

$$
\text { FORMAT ( IX, I5, 4FI5, 8) }
$$

$\mathrm{M}=\mathrm{N}+\mathrm{I}$

DO $42 \quad I=I, M$

$\mathrm{Y}(\mathrm{I})=\mathrm{AO} *(1-10 * *(-\mathrm{CO} *(\mathrm{Z}(\mathrm{I})+\mathrm{BO}))) * 10 * *(-\mathrm{PKO} *(\mathrm{Z}(\mathrm{I})+$ $+\mathrm{BO}) \star \star 2)$

$J=I+I$

$\mathrm{Y}(\mathrm{J})=\mathrm{AO} *(1-10 * *(-\mathrm{CO} *(\mathrm{Z}(\mathrm{J})+\mathrm{BO}))) * 10 * *(\mathrm{PKO} *(\mathrm{Z}(\mathrm{J})+\mathrm{BO})$ $\star \star 2$ )

$I F(\underline{Y}(J)-Y(I)) 20,20,42$

20 NRTTE $(3,200) I, J$

200 FORMAT (IX,'TROCA DE SINAL PARA I=I,I3,'E J=',I3) $X=Z(I)$

32

$\mathrm{Y} I=\mathrm{AO} *(1-10 * *(-\mathrm{CO} *(\mathrm{X}+\mathrm{BO}))) * 10 * *(-\mathrm{PKO} *(\mathrm{X}+\mathrm{BO}) * \star 2)$

$\mathrm{X}=\mathrm{X}+\mathrm{DE} \mathrm{LTA}$

$\mathrm{Y} 2=\mathrm{AO} *(1-10 * *(-\mathrm{CO} *(\mathrm{X}+\mathrm{BO}))) * 10 * *(-\mathrm{PKO} *(\mathrm{X}+\mathrm{BO}) * \star 2)$

$\mathrm{DIF}=\mathrm{Y} 2-\mathrm{Y} I$

WRITE $(3,202) Y 1, Y 2, X, D I F$

202 FOR:IAT $\left(I X, ' Y I=', F 15.8,{ }^{\prime} Y 2={ }^{\prime}, 15.8, ' X={ }^{\prime}, F 15.8\right.$, 'DIF=',F士5.8)

$\mathrm{TEST}=\mathrm{ABS}(\mathrm{DIF})$

IF (TEST-0.0000001) 11,11,50

$50 \operatorname{IF}(\mathrm{DIF}) 41,41,32$

$41 \quad \mathrm{X}=\mathrm{X}-\mathrm{DELTA}$

$\mathrm{DELTA}=\mathrm{DELTA} / 2$ 
GO TO 32

42 CONTINUE

II GO TO 1000

END

FEATURES SUPPORTED

ONE WORD INTEGERS

EXTENDED PRECISION

IOCS

CORE REQUI REMENTS FOR

COMMON 0 VARIABLES

16B PROGRAM

END OF COMPILATION

// XEQ 
69.

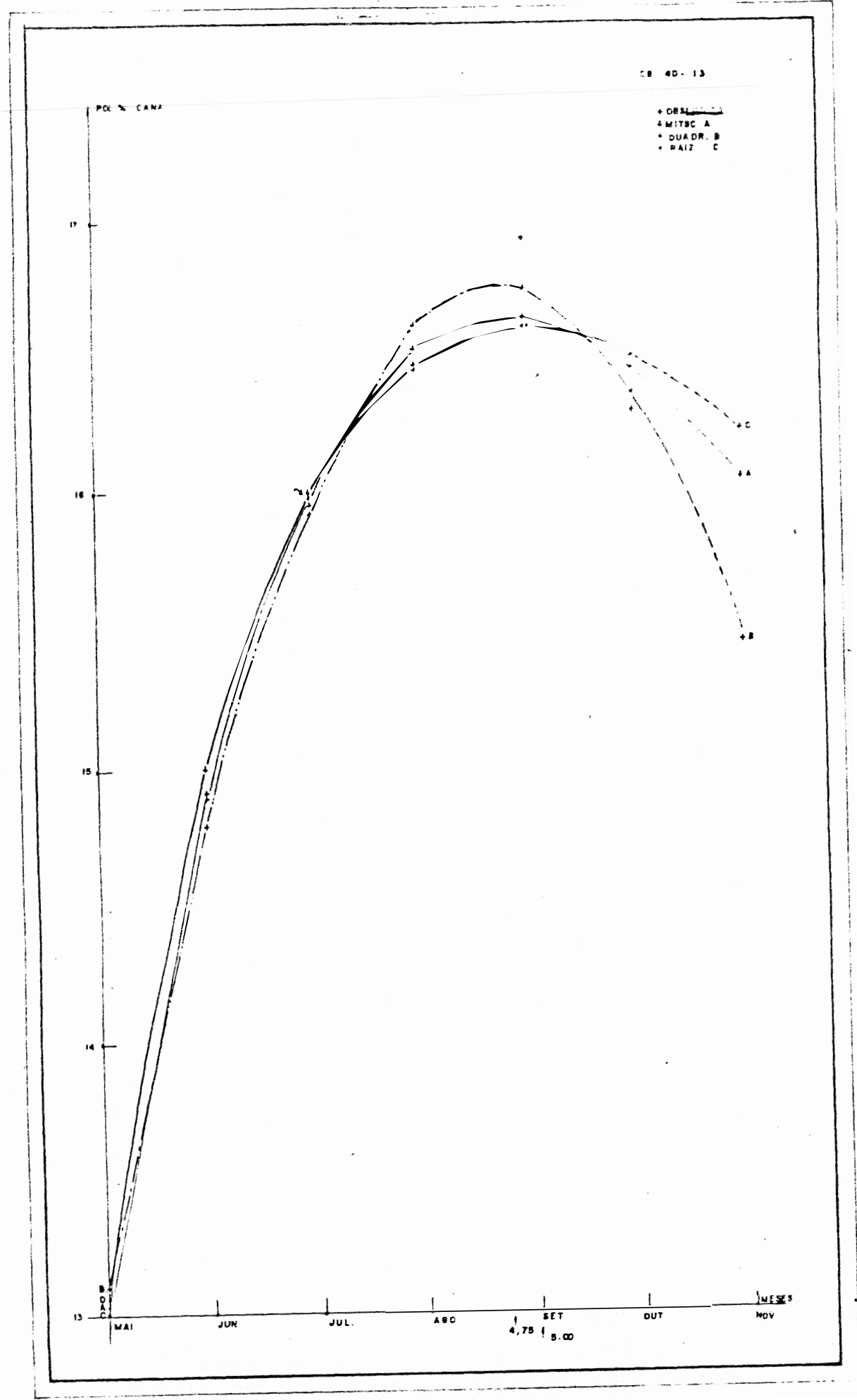




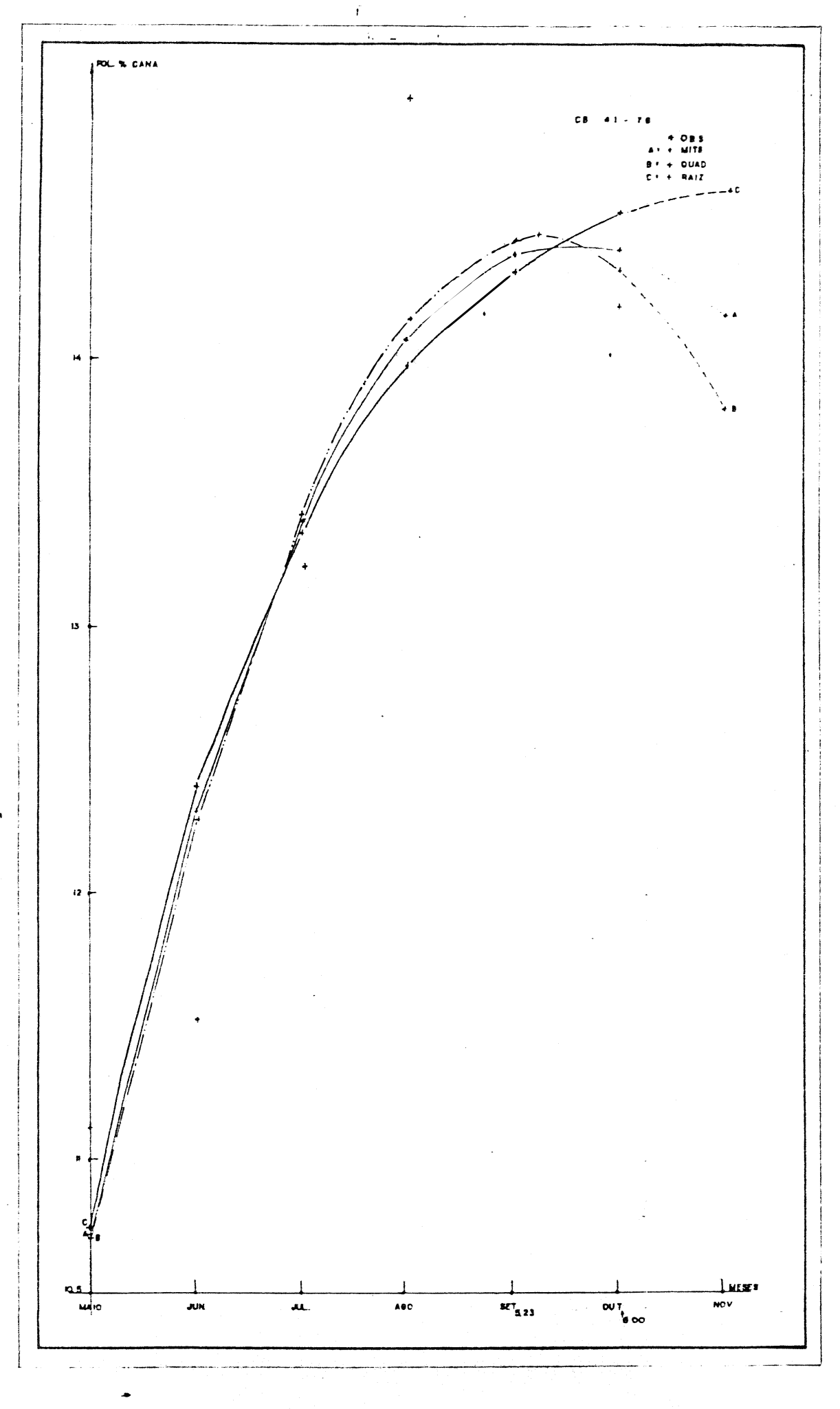




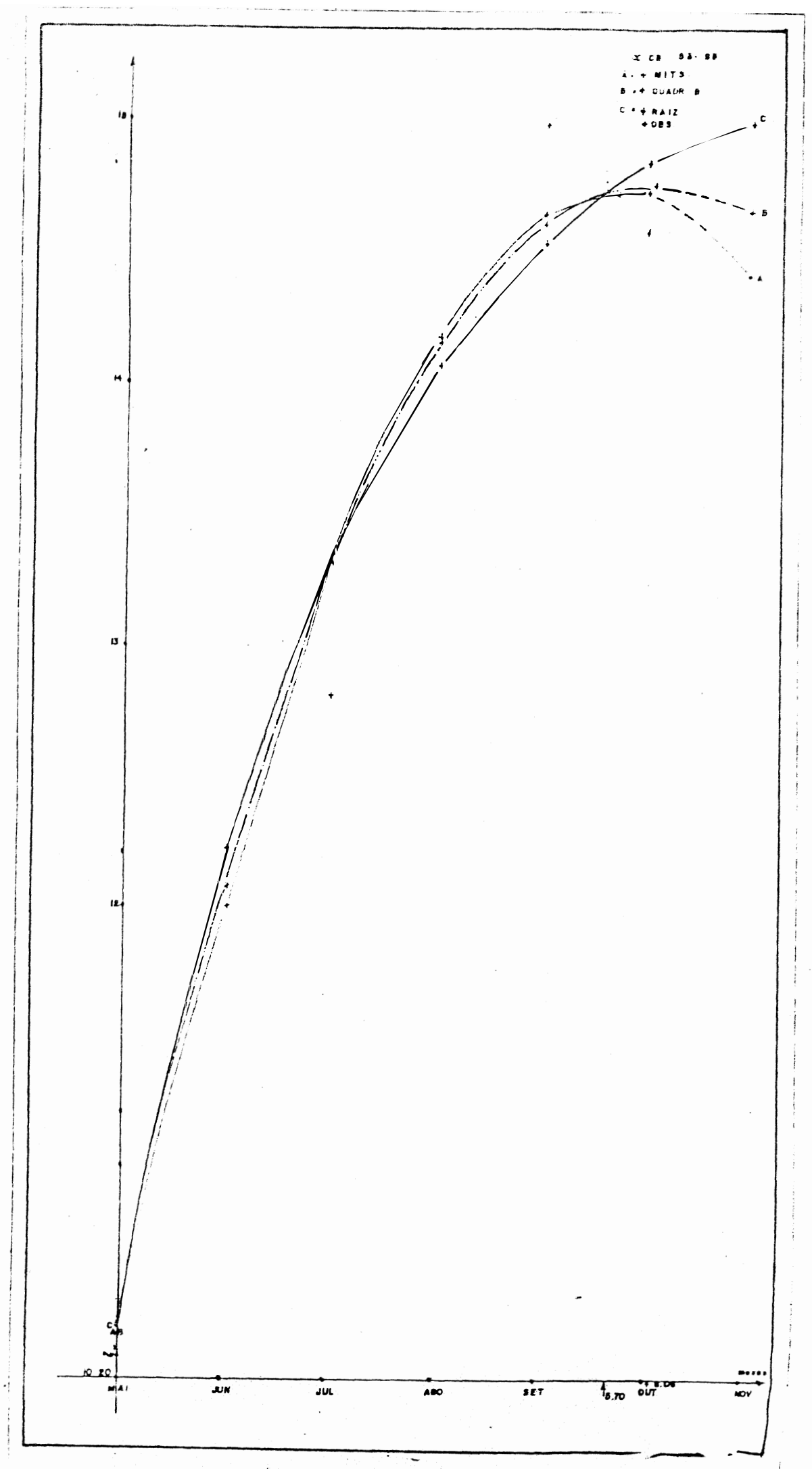


72.

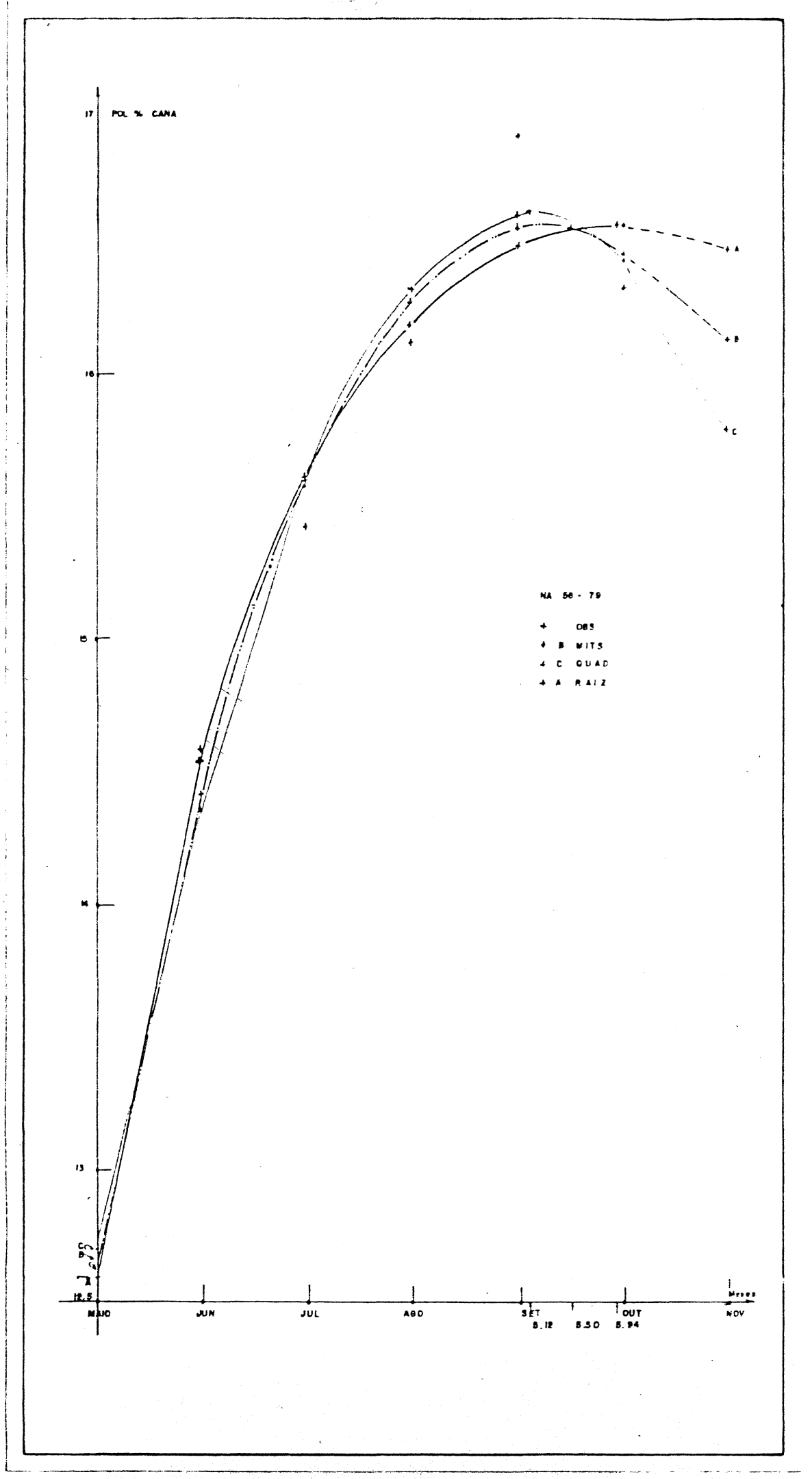


73.

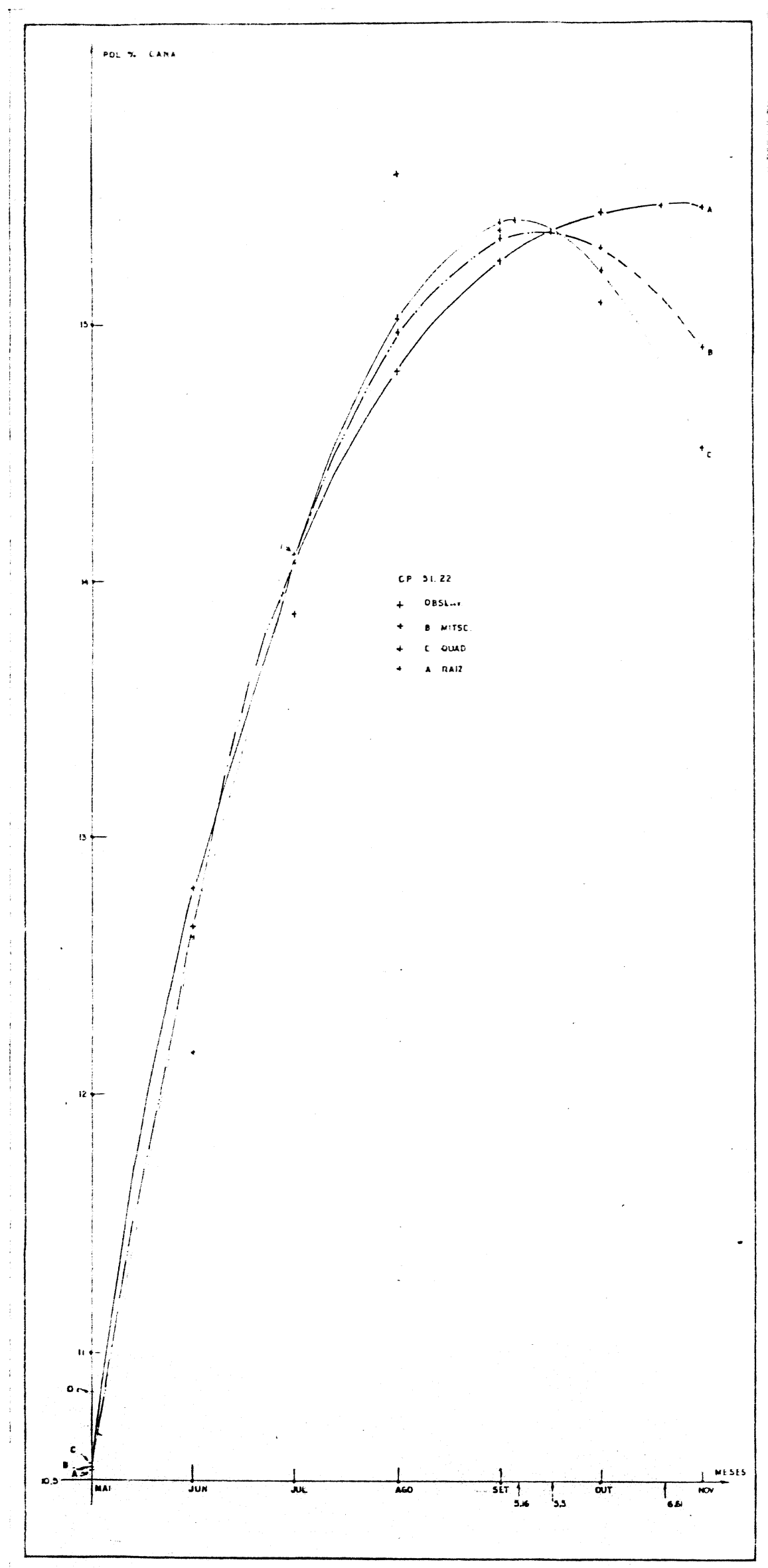




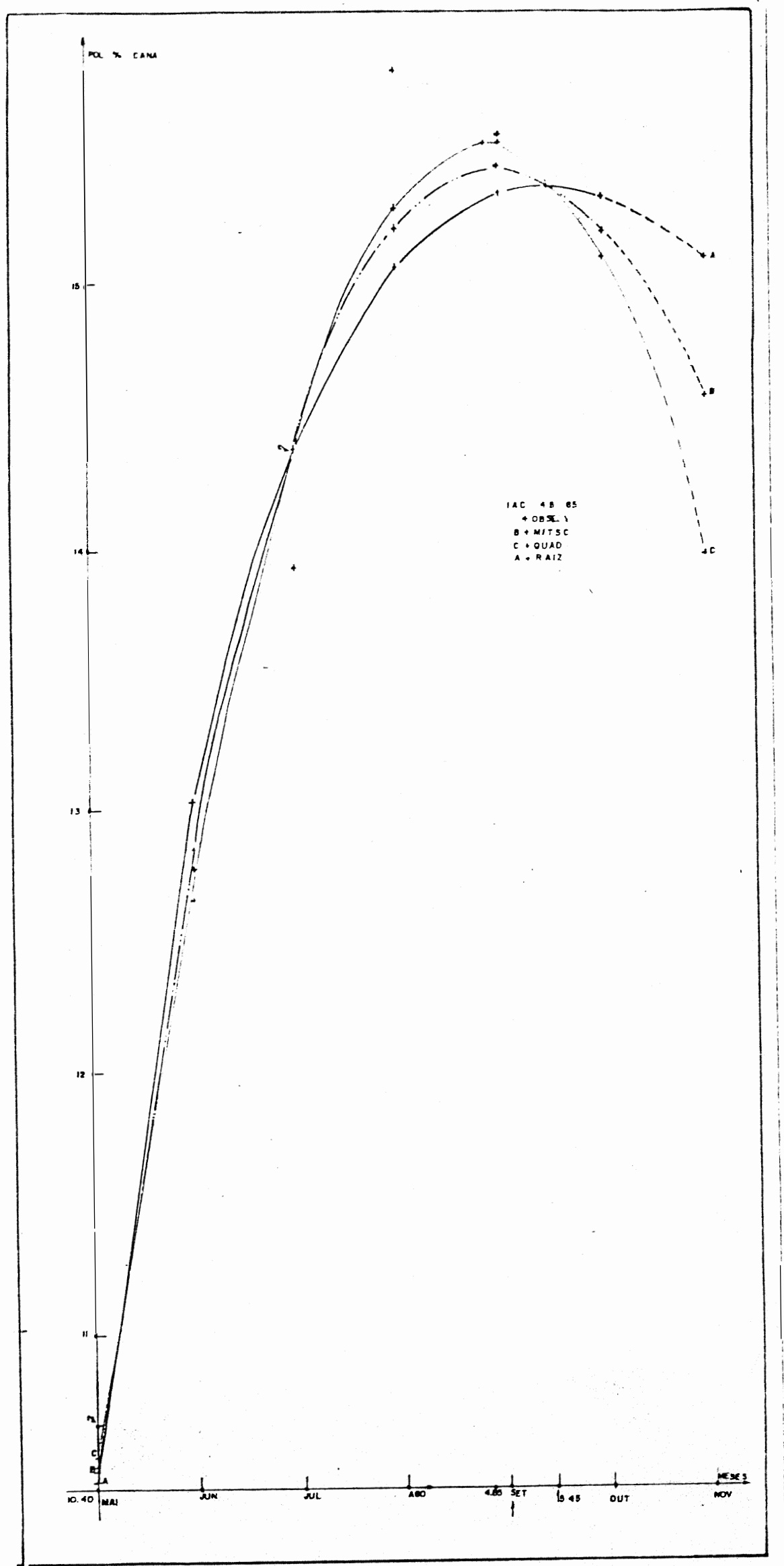

